\title{
A Four-legged Social Robot based on a Smartphone
}

\author{
A Thesis Presented to \\ The Faculty of the Computer Science Program \\ California State University Channel Islands \\ In (Partial) Fulfillment \\ of the Requirements for the Degree \\ Masters of Science in Computer Science
}

by

David Angelo Diano

August 2014 


\section{(C) 2014}

David Angelo Diano

ALL RIGHTS RESERVED 


\section{APPROVED FOR THE COMPUTER SCIENCE PROGRAM}
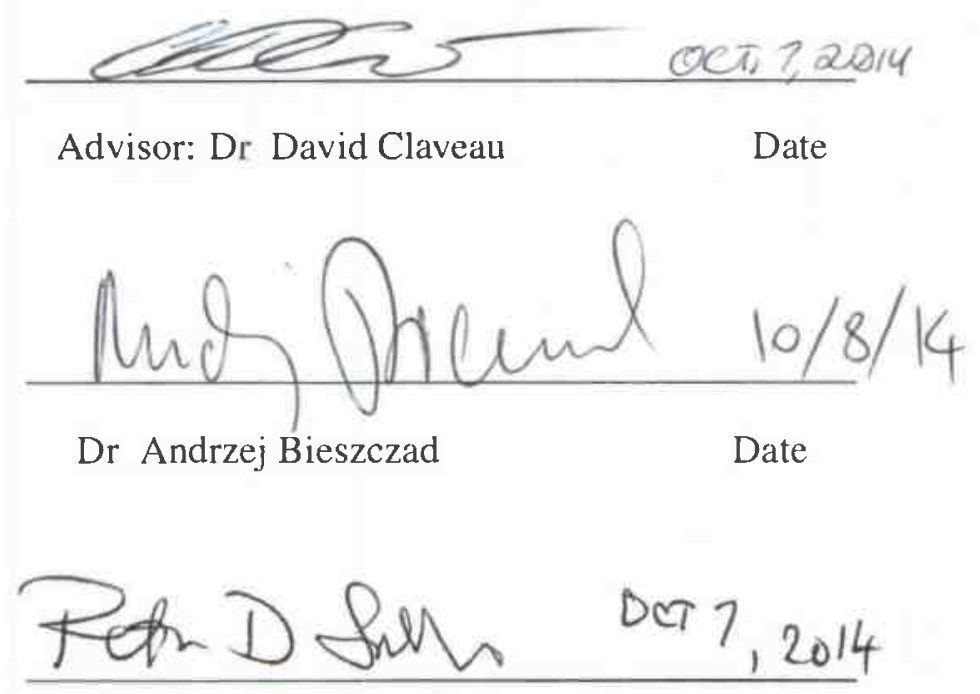

Dr Peter Smith Date

APPROVED FOR THE UNIVERSITY

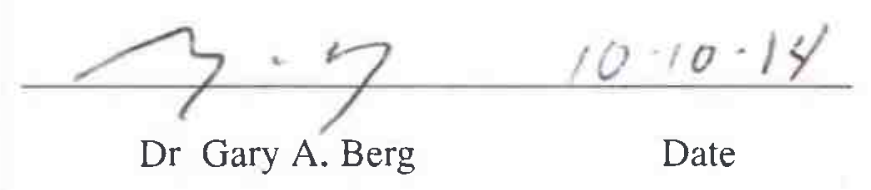




\section{Non-Exclusive Distribution License}

In order for California State University Channel Islands (CSUCI) to teproduce, translate and distribute your submission worldwide through the CSUCI Institutional Repository, your agreement to the following terms is necessary, The author(s) retain any copyright currently on the item as well as the ability to submit the item to publishers or other repositories.

By signing and submitting this license, you (the author(s) or copyright owner) grants to CSUCI the nonexclusive right to reproduce, translate (as defined below), and/or distribute your submission (including the abstract) worldwide in print and electronic format and in any medium, including but not limited to audio or video.

You agree that CSUCI may, without changing the content, translate the submission to any medium or format for the purpose of preservation.

You also agree that CSUCI may keep more than one copy of this submission for purposes of security, backup and preservation.

You represent that the submission is your original work, and that you have the right to grant the rights contained in this license. You also represent that your submission does not, to the best of your knowledge, infringe upon anyone's copyright. You also represent and warrant that the submission contains no libelous or other unlawful matter and makes no improper invasion of the privacy of any other person.

If the submission contains material for which you do not hold copyright, you represent that you have obtained the unrestricted permission of the copyright owner to grant CSUCI the rights required by this license, and that such third party owned material is clearly identified and acknowicdged within the text or content of the submission. You take full responsibility to obtain permission to use any material that is not your own. This permission must be granted to you before you sign this form.

IF THE SUBMISSION IS BASED UPON WORK THAT HAS BEEN SPONSORED OR SUPPORTED BY AN AGENCY OR ORGANIZATION OTHER THAN CSUCI, YOU REPRESENT THAT YOU HAVE FULFILLED ANY RIGHT OF REVIEW OR OTHER OBLIGATIONS REQUIRED BY SUCH CONTRACT OR AGREEMENT:

The CSUCI Institutional Repository will clearly identify your name(s) as the author(s) or owner(s) of the submission, and will not make any alteration, other than as allowed by this license, to your submission.

\section{A Four-legged Social Robot based on a Smartphone}

Title of Item

\section{Social robot, Smartphone, Legged robot, Robot postures}

3 to 5 keywords or phrases to describe item

\section{David Angelo Diano}

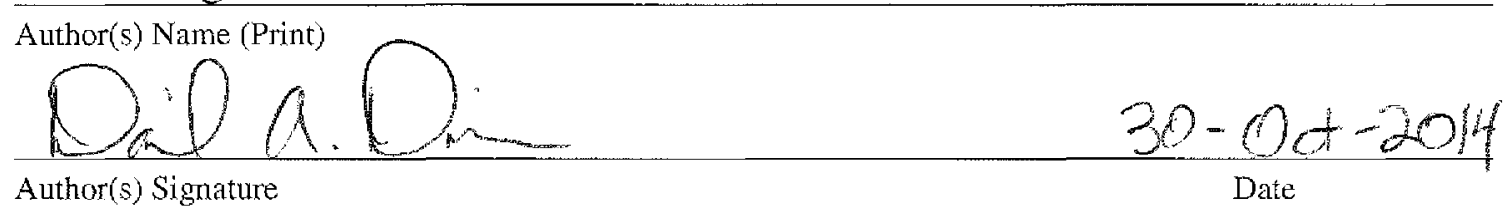

This is a permitted, modified version of the Non-exclusive Distribution License from MIT Libranies and the University of Kansas. 


\title{
A Four-legged Social Robot based on a Smartphone
}

\author{
by \\ David Angelo Diano \\ Computer Science Program \\ California State University Channel Islands
}

\begin{abstract}
This thesis presents a simple four-legged robot platform that can transform a smartphone or tablet into an autonomous walking social robot. Smartphones are already designed to be easy to interact with and to assist humans in everyday activities. They are equipped with impressive computational resources and powerful sensors and can serve as a controller for a robot. By giving them four legs they have the added ability to walk about our environment and to express themselves using postures and body language. An example of such a fourlegged robot is described in this paper along with a preliminary exploration of its capabilities as a social robot. Simple walking and posturing are demonstrated in ways that show how such a robot can better play the role of companion or assistant in the office or home. The platform is designed and built using inexpensive off-the-shelf components and can serve as an affordable development system for students and practitioners who wish to study social robotics.
\end{abstract}




\section{Acknowledgements}

First and foremost I would like to thank my advisor Dr. David Claveau who has supported me throughout my thesis work. Without his patience, supervision, and constant help this paper would not have been possible. In addition, I would like to thank Dr. Andrzej Bieszczad and the Channel Islands Computer Science faculty who have supported me throughout all my studies at the university. A special thanks to my parents for their contributions, advice, and continued support. Words cannot express how grateful I am for all of the sacrifices that you've made on my behalf. 


\section{Table of Contents}

1 Introduction $\quad 11$

2 Background and Related Work $\quad 14$

2.1 Introduction to Social Robots . . . . . . . . . . . . . . . . . . . . 14

2.2 The Smartphone as a Personal Assistant/Companion . . . . . . . . . 15

2.3 Robots Based On Smartphones . . . . . . . . . . . . . 17

2.4 The Need for Legs . . . . . . . . . . . . . . . . . . . . 19

3 The Robot Platform $\quad 21$

3.1 Robot Body Design . . . . . . . . . . . . . . . . 21

3.2 Robot System Level Design . . . . . . . . . . . . . . . 25

3.3 Robot Software Design ........................ 27

4 Implementation $\quad 29$

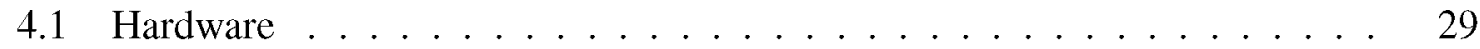

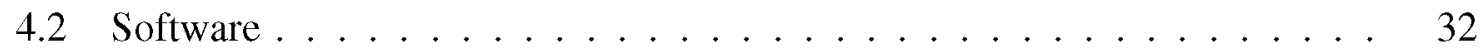

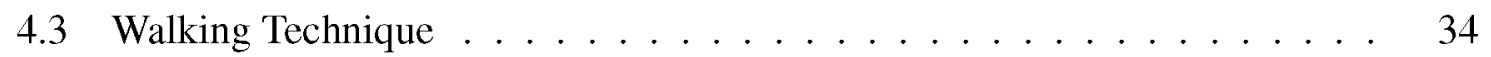

5 Experiments and Results $\quad 37$

5.1 Good Morning Robot! . . . . . . . . . . . . . . 37

5.2 Robot Body Postures for Conversation . . . . . . . . . . . . 37 
6 Conclusion $\quad 41$

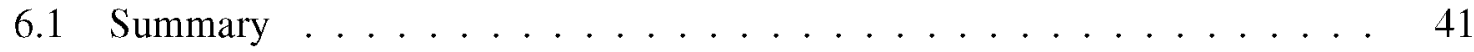

6.2 Future Work . . . . . . . . . . . . . . . . . . . . 41

$\begin{array}{ll}\text { References } & 43\end{array}$ 


\section{List of Figures}

Figure 1.1 Commercially available smartphone robots include (a) Romo [1] and

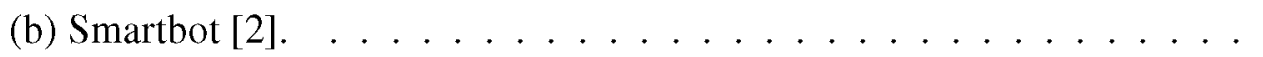

Figure 2.1 Second generation Grey Walter turtle that is held in the Smithsonian Institution. . . . . . . . . . . . . . . . .

Figure 2.2 Smartphone Digital Personal Assistants (a) Siri for the Apple iPhone and (b) Google Now for Android. . . . . . . . . . . . . . 17

Figure 2.3 MeBot mimics the head and arm movements of user. . . . . . . 18

Figure 2.4 BigDog robot can walk across rubble, climb hiking trails and walk in snow and water. . . . . . . . . . . . . . . .

Figure 3.1 Examples of a one-legged hopping robot and a two-legged walking

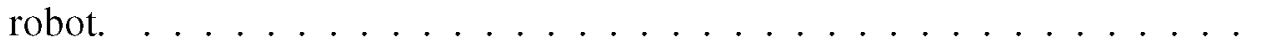

Figure 3.2 The RoMeLa STriDER has a unique tripedal locomotion that is stable and agile while walking. . . . . . . . . . . . 23

Figure 3.3 Insects such as spiders are usually chosen as models for hexapod robots. 24

Figure 3.4 The base and legs of the robot platform. . . . . . . . . . 26

Figure 3.5 System block diagram for the robot platform. . . . . . . . . . 27

Figure 3.6 State transition diagram for robot application. . . . . . . . . 28

Figure 3.7 Execution proccess for robot application. . . . . . . . . . 28

Figure 4.1 Final construction of smartphone walking robot. . . . . . . . 30 
Figure 4.2 Schematic showing the connections for each robot component. . . . 31

Figure 4.3 Software stack for the robot platform. . . . . . . . . . . 33

Figure 4.4 Walking gait of a dog (a) was used as a model for robot leg movement

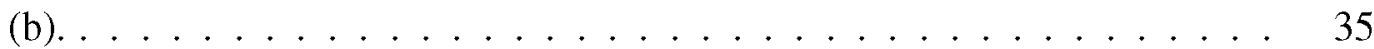

Figure 5.1 https://www.youtube.com/watch?v=ErMmKLlDkp4 . . . . . 38

Figure 5.2 Example postures (a) bowing action as if listening, (b) front of robot nods if a command is understood, (c) robot rocks forward and backwards if confused, and (d) waves front arm waving goodbye. . . . . . . . . .

Figure 5.3 Survey results for robot postures (intended response is circled for each posture). Posture 1 is 'ready to listen', posture 2 is 'understand', posture 3 is 'confused', and posture 4 is 'waving goodbye'. . . . . . . . . . . . . 


\section{Introduction}

Powerful mobile devices such as smartphones and tablets have become commonplace items. These devices have been designed to allow humans to interact with them in rich ways using visual display, touch and voice. However, they remain devices that must be carried around, locked in a body without mobility despite the fact that they contain the computational resources and the sensors needed to control an articulated robot body. Even the least expensive of these devices contain cameras, accelerometers and GPS. An internet search for "smartphone robot' is likely to find some interesting examples such as Romo [1], a programmable robot companion that uses an Apple iPhone as a controller. Romo can respond to humans and roam around a desktop using tracked wheel locomotion. Another example is the SmartBot [2], a small robot that can use Android or iOS-based smartphones to control its behavior. Both are shown in Figure 1.1. While these robots add mobility to a smartphone, they are limited in their ability to move. They cannot move in ways that dogs, cats and other four-legged creatures can. We have become very familiar and comfortable with four-legged creatures and we easily understand their body language. If we could plug our smartphone into a fourlegged body, it would allow the device to embody an assistant more closely or perhaps a robot 'familiar' like a magician's black cat. Your smartphone could now climb onto your shoulder and alert you by whispering in your ear, perhaps helping you to cross a street if you have a visual impairment. It could also use a form of body language to express itself more clearly. Such a robot would be a 'personal' or 'social' robot rather than a traditional industrial robot.

Social robots must learn to work together with a user to perform tasks, learn new skills, 


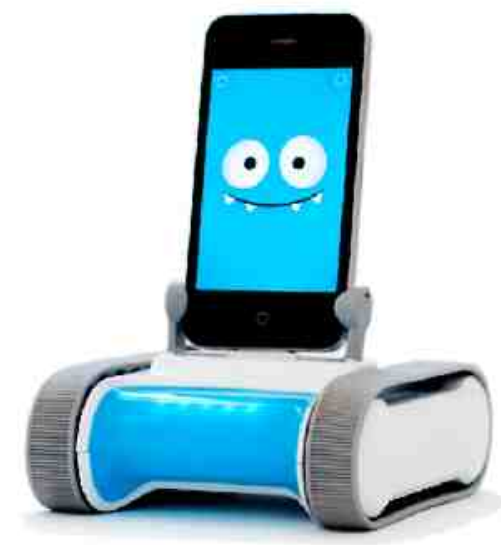

(a) Romo

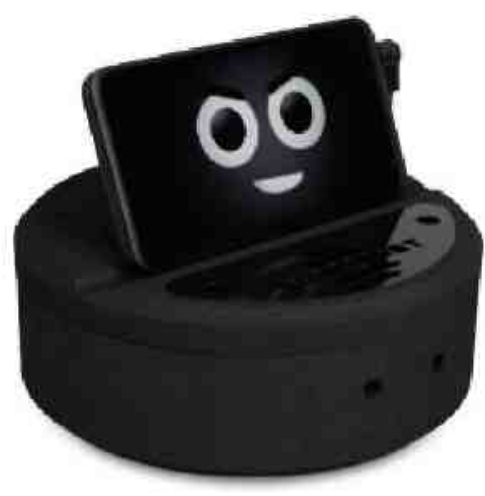

(b) Smartbot

Figure 1.1: Commercially available smartphone robots include (a) Romo [1] and (b) Smartbot [2].

and exchange information with one another in order to become a member of society. There has been much research devoted to human-robot interaction and how it can be applied to the field of social robotics. Cynthia Breazeal, director of the Personal Robots Group at the MIT Media Laboratory, has described these robots as a "really intriguing social technology" [14]. Even in manufacturing, a company called Rethink Robots [15] has created a new humanshaped robot that can work alongside people to help increase productivity for mundane tasks. As these types of robots become more common in the workplace environment, there will be a need for social interaction with either human or robot coworkers. Robot workers that can learn new tasks by social interaction would now be able to save the time and money needed to reprogram them for another purpose.

In some ways these changes in the field of robotics can be compared to the beginning of the personal computer revolution in the 1970 s. It is expected that people will have their own robots in the workplace or at home and have them operating in their personal lives in new and exciting ways. The introduction of low-cost robotic kits and improved software can help students, researchers and practitioners to experiment in the field of robotics and to develop exciting new commercial applications where robots could interact with people in more engaging ways. Inexpensive controller boards, sensors and motors can be quickly 
assembled into a mobile robot that can perform both useful and entertaining functions and soon we will start to see a new generation of robotics emerge.

In this thesis I present a simple four-legged robot platform that can transform a smartphone or tablet into an autonomous walking robot. A smartphone can be simply 'plugged into' the body and then control its own motion. An important aspect of the design is that only inexpensive off-the-shelf components are needed along with open source software. This makes it a convenient platform for students who want to explore social robotics. Just about every university student has a smartphone or tablet and soon even young children will have such devices. This opens some exciting possibilities in education since students can simply plug their device into the robot platform and program new behaviors. This thesis presents some simple examples of walking and posturing behaviors. Chapter 2 discusses some related work and following that the smartphone robot platform is described in chapter 3. Implementation and preliminary results are presented in chapters 4 and 5 with an informal survey that was performed to explore the effectiveness and reception by an average smartphone user. 


\section{Background and Related Work}

\subsection{Introduction to Social Robots}

The field of social robotics was first introduced by neurophysiologist and roboticist William Grey Walter around 1940 to 1950 . He created three-wheeled, turtle like, robots that would interact in a social manner by exploring their environment and responding to light stimulus [20] (Figure 2.1). The research and experiments performed by Grey Walter on artificial intelligence would eventually lead to the emulation of social concepts in nature like insect swarm behavior and natural language communication. Robotics experienced another breakthrough in the late 1980s and early 1990s as new concepts developed in artificial intelligence were incorporated into more advanced robot bodies leading to the field of behavior-based robotics [24]. Based on this, researchers began building robots with complex behavior systems that could learn to be digital assistants that try to exhibit behavior indistinguishable from a human.

For humans and animals, participating in social interaction comes naturally and it can sometimes become difficult to explain some of these social concepts to a robot. Furthermore, a robot programmed to be social must also learn how to adapt as languages and gestures evolve with new generations introduced. A socially intelligent robot can quickly become a complicated system and there is much information about social robot concepts such as artificial intelligence, human-robot interaction, and developmental psychology that is beyond the scope of this thesis. Designing a social robot that can be used by consumers presents a unique challenge of getting the robot to interact in a natural way while supporting a range of 


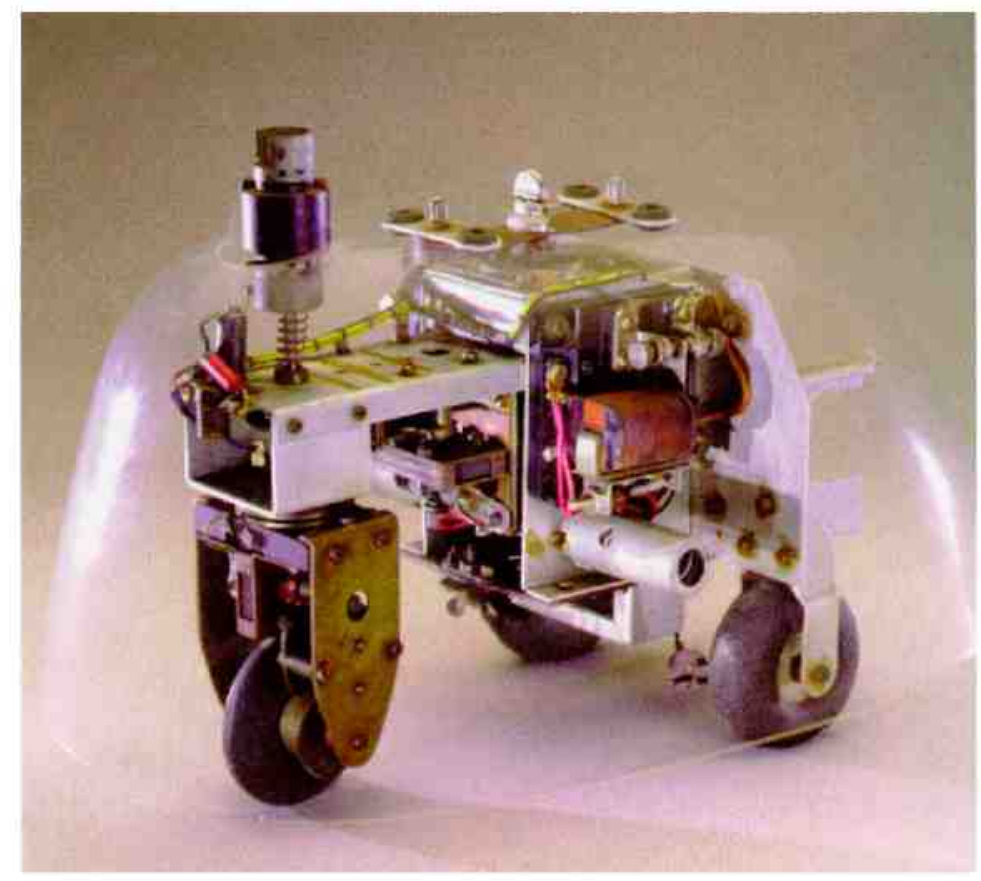

Figure 2.1: Second generation Grey Walter turtle that is held in the Smithsonian Institution.

user qualities such as gender, ages and diverse cultural backgrounds. The consumers must be able to teach the robot new skills, similar to a child, and in turn be treated as a unique individual through the robot's natural interaction. Breazeal has been working on robots for the past decade and worked on a number of robot projects that deal with social interaction. In a 2010 TED Talk called "The Rise of Personal Robots" [14], she explains how people enjoy socializing with robots more than getting information from a computer screen. A study over several weeks found that people would spend more time interacting with a social robot than a touch-screen computer interface even though the quality of information that they provided was identical.

\subsection{The Smartphone as a Personal Assistant/Companion}

Mobile phones and tablets are becoming some of the most common possessions and people who have such devices are usually interacting with them on a daily basis. The definition of a smartphone is a device that combines the features of a mobile phone with the functions 
of a computer and other consumer devices, including personal information managers, media players, GPS navigation, and digital cameras. Smartphones also contain various sensors that can be used to take motion (accelerometers, gyroscopes), environmental (barometers, thermometers) and position (orientation, magnetometers) measurements. Since all of these features can fit in the palm of your hand, it is no wonder why people spend much of their time on their phones looking up information and using them for entertainment and social interaction.

Most modern smartphones also have a type of digital personal assistant which users can interact with using natural language. Apple's Siri [16] is an example of an intelligent personal assistant integrated into iOS devices that can answer questions, perform requested actions or make recommendations based on the information that a user provides. Google also provides a personal assistant application called Google Now [17] that is similar to Apple's Siri assistant. Figure 2.2 shows the two interfaces from Google and Apple side by side. Besides answering questions using a natural language user interface, Google Now will also try to show relevant information to the user and is able to predict what the user is interested in based on search habits. Both of these personal assistant services are starting to make users feel comfortable interacting with their smartphones in a helpful yet social way. Audio feedback is a great social tool for robots but it still lacks some of the other components humans use to carry on a conversation such as visual cues and emotion.

The personal nature of our relationship with smartphones and tablets is a topic of current exploration [3]. These devices now contain everything from our memories of happy and sad experiences to our preferences in music and movies, but our physical relationship with these devices is rather limited. Since visual interaction is confined to the display we can think of these devices as 'virtually embodied'. Studies have shown that a 'physically embodied' robot that can move its own body is more engaging [4][5][6]. These studies have also shown that physical embodiment leads to more feelings of enjoyment, trust and respect toward the robot. An example of a socially expressive robot based on a smartphone is MeBot [7]. This 


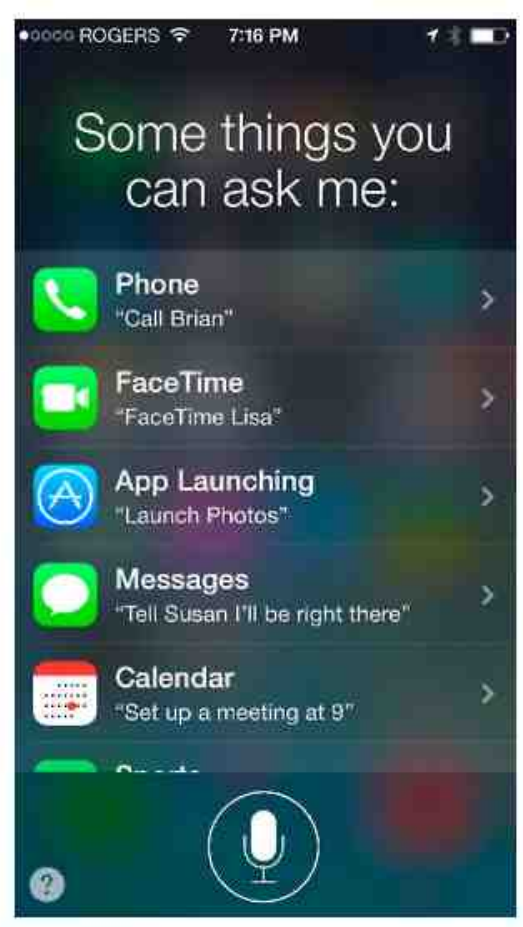

(a) Siri on iOS 7

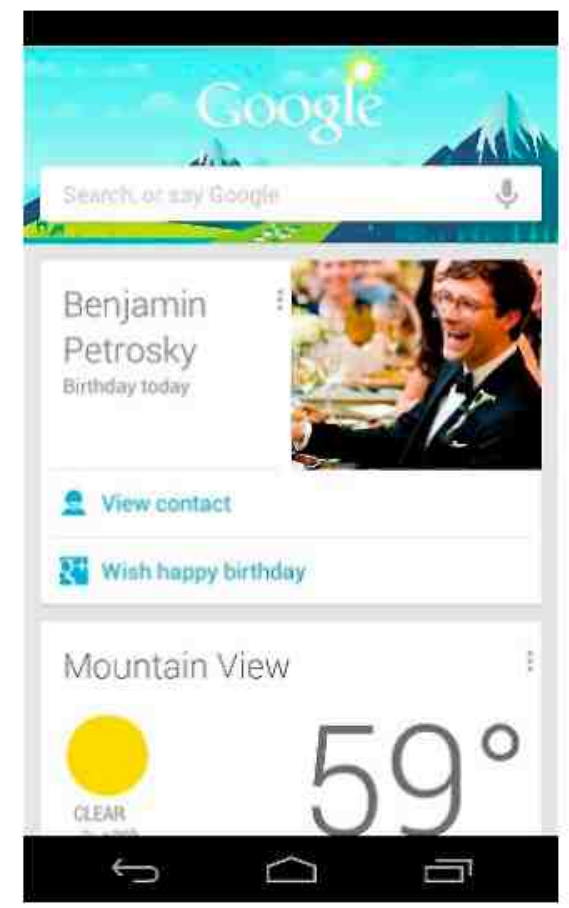

(b) Google Now on Android

Figure 2, 2: Smartphone Digital Personal Assistants (a) Siri for the Apple iPhone and (b) Google Now for Android.

is a telepresence robot that allows an operator to express nonverbal behavior such as hand and head gestures. It combines video and audio of the remote operator's face with mechanical arms and wheeled desktop mobility (Figure 2.3). The MeBot project is a significant contribution to the field of social robotics that not only introduces people to a more engaging interaction using their smartphone but also opens the possibilities to robots based on mobile devices.

\subsection{Robots Based On Smartphones}

There are several examples of mobile robots based on smartphones that bear some similarity to the work in this thesis but they have generally been wheeled robots and have not addressed the use of a legged body or social interaction issues. A couple of options are available when choosing an interface that connects the smartphone to a robot body. A popular choice is an Android-based smartphone directly connected to a IOIO (or the newer IOIO-OTG), 

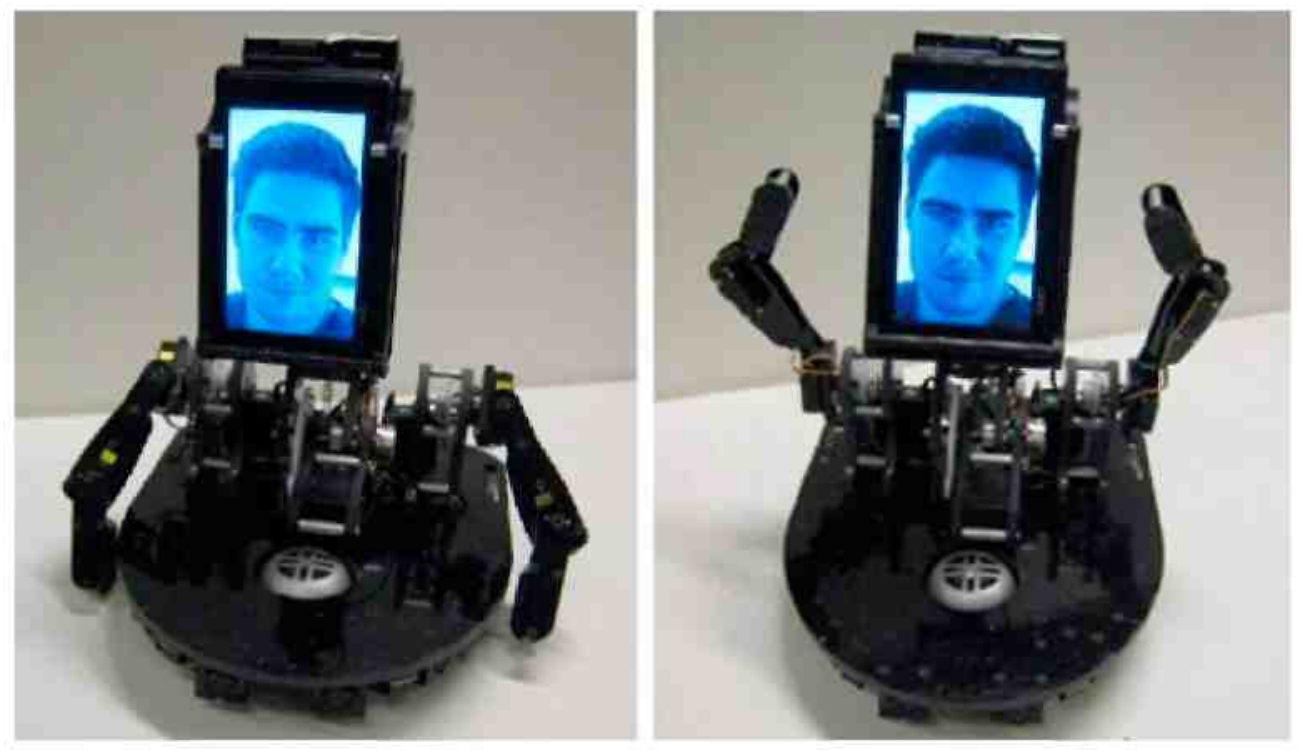

Figure 2,3: MeBot mimics the head and arm movements of user.

pronounced "yo-yo", circuit board that controls servos and other sensors [25]. The IOIO board can be connected to the smartphone via Bluetooth or USB and can read values from digital/analog sensors using the Java API provided. For example, an Android smartphone can be programmed to send commands through a IOIO board that is connected to an inexpensive remote controlled car [8][9]. The smartphone can read sensors, like the built-in GPS or an infrared sensor connected to the IOIO, and send commands to the servos and electronic speed controller to navigate the car. Another option for building a smartphone robot is using the LEGO Mindstorms system [10], which is used in schools and universities because of its affordable yet flexible building options.

Many robotic projects can use a smartphone and an interface board to reduce overall cost, however, the body still needs to be purchased and can cost an additional $\$ 250$ to over $\$ 500$, which sometimes does not include essential electronics such as a controller board or sensors. For example, the newest LEGO Mindstorms EV3 robot kit costs around $\$ 350$ for 3 servos and about 4 sensors. It provides a full robot body that allows you to experiment with some humanoid features such as grabbing objects with the robotic arm and using the camera for a vision system that can detect motion and colors. It can also perform simple navigation tasks 
using the distance sensor but still becomes less attractive when trying to develop a robot that can be programmed with a complex social behavior system. Even though prices for constructing robots will probably decrease with time, we would still like to consider the cost of building our walking robot as a comparison with other commercial robot kits that seem limited.

\subsection{The Need for Legs}

While wheels are fine for smooth desktop-like surfaces, legs are needed for unstructured surfaces, for instance cluttered desktops and lumpy beds. They also allow the robot to assume expressive postures and poses. Several projects have demonstrated that a smartphone can control a wheeled robot such as an off-the-shelf R/C hobby grade vehicle, but we would like to see more projects use a smartphone connected to legged robots. Legged robots are a very active research topic and we will go over some options when choosing a robot with legs in section 3.1. A prominent example is the Boston Dynamics BigDog robot [11], shown in Figure 2.4, which can achieve animal-like mobility on uneven terrain with a focus on searchand-rescue applications. The robot is very impressive but is very sophisticated and expensive. The robot platform in this paper is an attempt to combine the mobility and expressiveness of legs in an open and affordable platform that can be used by students and researchers to explore physically embodied social robotics.

Creating a walking robot is very challenging, considering the high cost of building one and the time it takes to design the leg physics or develop more human-like features. An important part of designing a walking robot is the leg locomotion. The walking mannerism is significantly dependent on the number of legs which also determines how much effort will go into the stability of your robot. More legs on a walking robot means more stability but this also has tradeoffs. For instance, robots with 4-6 legs are more stable but are considered less efficient at walking when considering the amount of resources (cost per leg, energy requirements) that are required for the leg movement pattern. Companies like Google are investing 

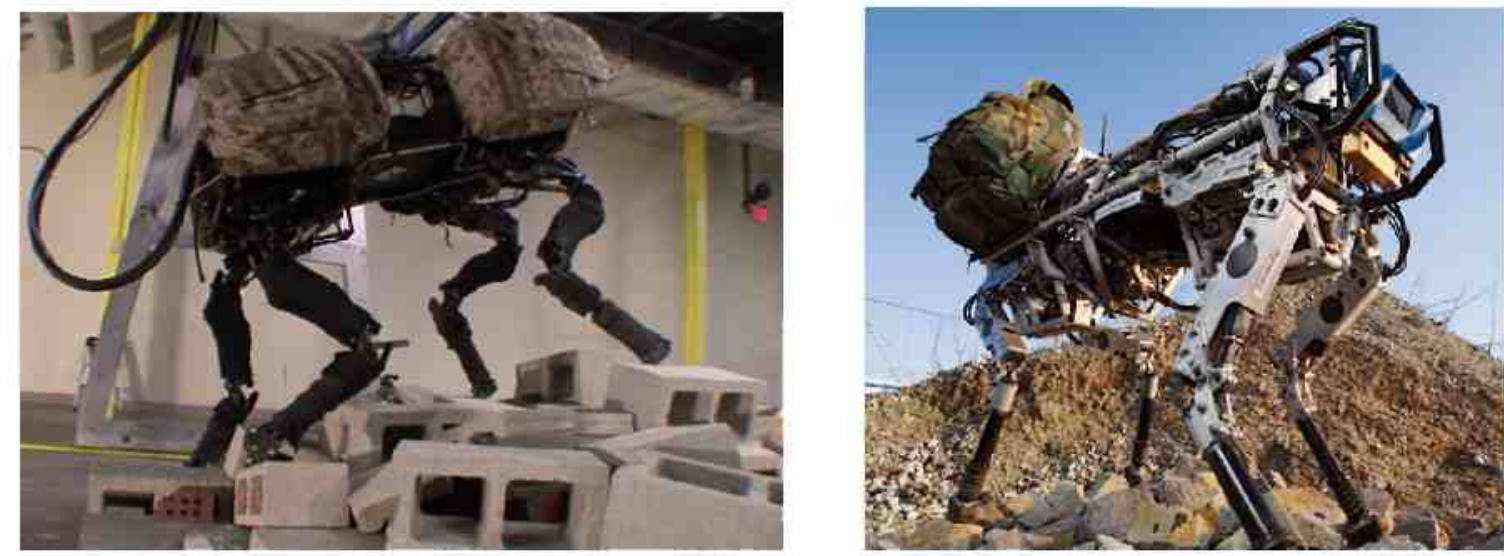

Figure 24: BigDog robot can walk across rubble, climb hiking trails and walk in snow and water.

in walking robots that can be used to respond to disasters such as earthquakes, so providing a platform that allows universities and companies to rapidly experiment with walking robotic concepts can help this field progress. The new platform presented in this thesis will not only accommodate educational purposes for a wide range of ages but is also flexible enough for commercial products in the near future. The next chapter looks at designing a walking four-legged robot that can serve as the first step in achieving a walking social robot. 


\section{The Robot Platform}

This section describes the design and implementation of the robot platform. The design had to meet the following basic requirements:

1. The platform should have four legs similar to a dog or cat.

2. The platform should be comparable in size and weight to a smartphone.

3. The platform should have an interface that allows a smartphone to be easily plugged into it.

4. The platform should allow for easy programming of behaviors.

\subsection{Robot Body Design}

As discussed in the previous chapter, leg locomotion is an important factor when designing a walking robot. Before a walking technique can be established, there must be a decision about how many legs to use, which ultimately depends on the functionality of the robot. The following sub-sections present some considerations when it comes to deciding the number of legs needed for a robot platform.

\section{A. One-legged robot}

One-legged robots are mainly designed to be hopping robots which require a great deal of balance. They need less building materials because most of the robot functionality focuses on balance and reliability. A robot with one leg can also help provide a design blueprint for another project should the requirements call for multiple legs to work together. A 3D OneLeg Hopper, shown in Figure 3.1(a), was developed at MIT to demonstrate how a robot with 


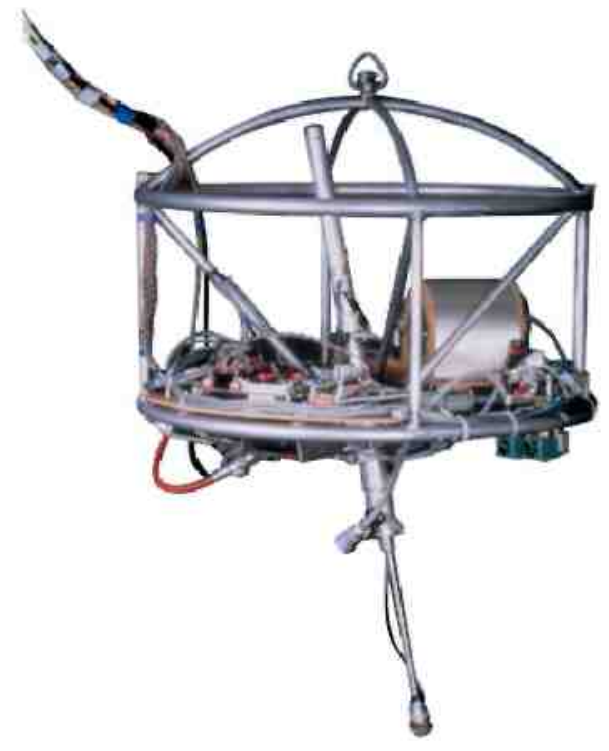

(a) MIT's 3D One-Leg Hopper robot

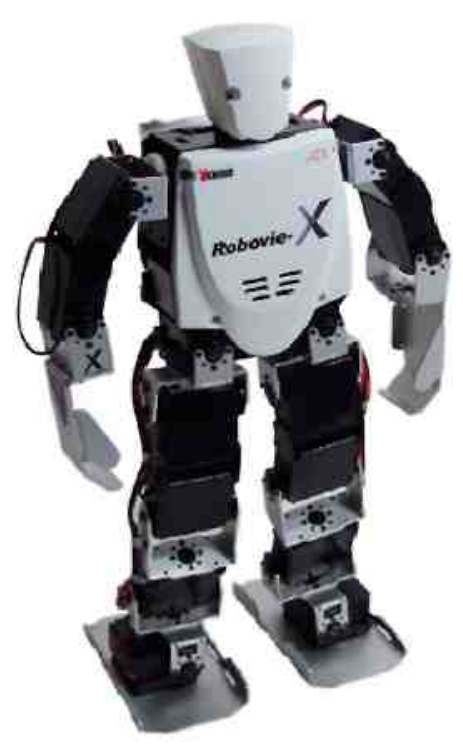

(b) Robovie-X by Vstone Co.,Ltd.

Figure 3.1: Examples of a one-legged hopping robot and a two-legged walking robot.

one leg can keep balance [19].

\section{B. Two-legged robot (biped)}

Robotics is a constant effort to emulate natural phenomena, and human-like motion and behavior is probably one of the most commonly researched areas. Bipeds, or humanoids like the one in Figure 3.1(b), can be designed with just a walking torso or have a full upper body that looks around and interacts with objects. Most development time is consumed on algorithms and data extraction that will keep the payload of a two-legged robot stable while it walks around or performs a task in the environment. Considering that humans put most of their pressure on the feet and for most types of humanoids it is no different, careful planning and sometimes the use of expensive materials are required to handle the weight of that pressure. Other important topics that are included in the development of humanoid robotics are artificial intelligence, joint modeling, object detection and avoidance, and cognitive development.

\section{Three-legged robot (tripod)}


Unfortunately there is very little research being done on tripod robots even though they are theoretically more stable than a biped. Since most robotic projects are based on the movement of an animal or insect, the concept of three legged locomotion can be difficult to imagine. Researchers at Virginia Tech have conceptualized a way that a tripod, shown in Figure 3.2, might walk. Two of the robots legs stabilize the weight while the third leg swings up between the two other legs giving it great movement flexibility. More information can be found on the RoMeLa website [18].

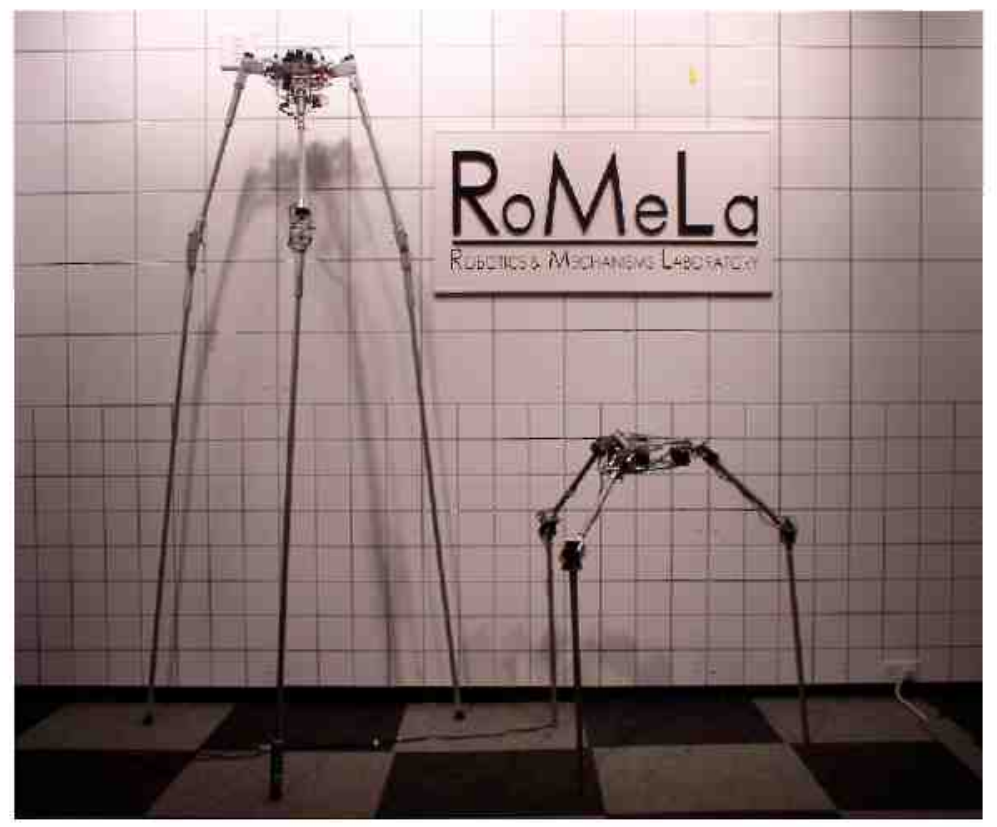

Figure 3.2: The RoMeLa STriDER has a unique tripedal locomotion that is stable and agile while walking.

\section{Four-legged robot (quadruped)}

Since quadruped robots offer a good mix of stability, freedom of movement, and speed, they probably are one of the easiest projects to work with. Most of the leg locomotion is modeled after four-legged animals so there is no need to try and develop a new physics model. Four legged robots are also simpler to build since minimally only 4 leg joints and a flat body to carry the electronics is needed. Boston Dynamics is one of many companies specializing in quadruped robots like the BigDog robot that was shown in Figure 2.4. Reaching up to $28 \mathrm{mph}(45 \mathrm{kpm})$ or carry up to 400 pounds of gear while walking up rough terrain, 

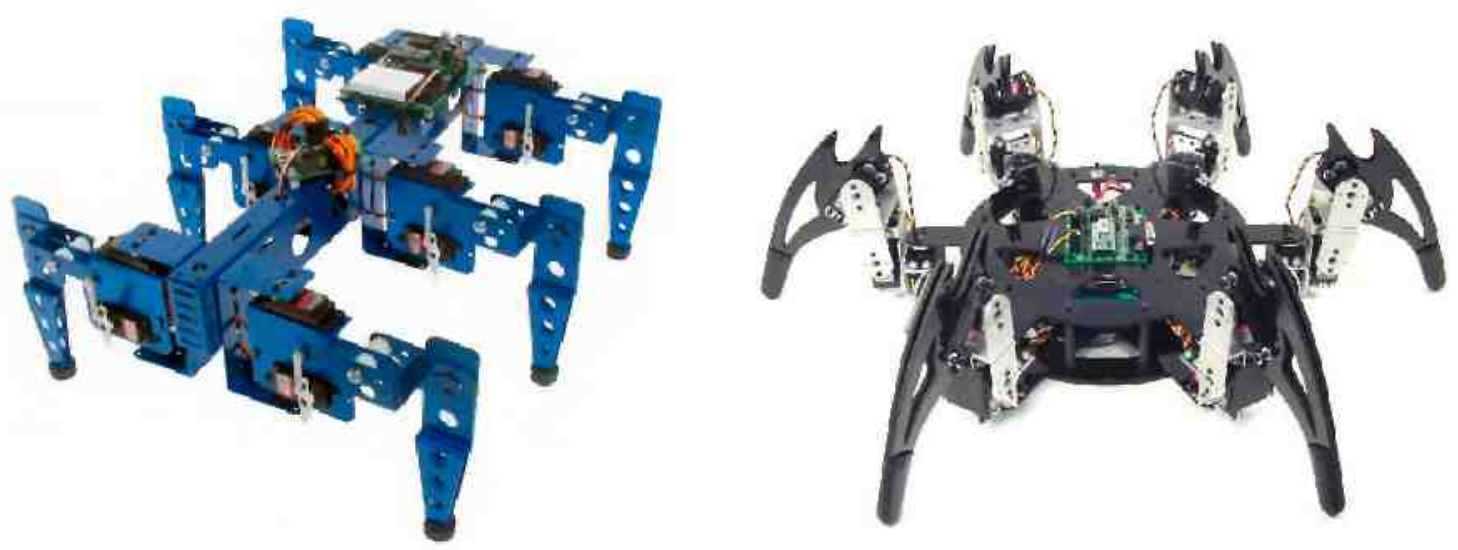

Figure 3.3: Insects such as spiders are usually chosen as models for hexapod robots.

these four-legged robots have a promising future for being useful in various tasks.

\section{E. Six-legged robot (hexapod)}

Hexapods and robots with more than six legs have a greater amount of stability but they also require more moving parts which increases the demand for more energy from the robot. Most of these robots mimic the movement of a spider or some other insect, which involve the coordination of all legs working together to move the robot. These robots can have more flexibility and smoother gestures by either crawling one leg at a time or alternating two or more legs at a time. Of course, the cost of building a robot dramatically increases as more servos and sensors are needed for steady movement. An example is the T8 project from Robugtix. It has 26 servo motors working together to give the octopod eerie life-like movements. Some examples of hexapod robots are in Figure 3.3.

\section{F. The robot in this thesis}

The goal of the walking robot in this thesis is to be simple and inexpensive but still have the ability to perform natural postures and exhibit personality and character. As such, the decision was made to use four legs. The main body of the platform, shown in Figure 3.4, is designed as a simple rectangular base made of a stiff material such as lightweight wood or plastic. Four legs are attached to the bottom of this base and a smartphone can be placed 
face up on top of it. The legs were designed to each have two revolute joints of 1-DOF. This is sufficient for simple walking gaits and simple postures and makes the legs very easy to design. In fact, each leg only used one 'femur' and used the servo-motor's body as the endeffector that touches the ground. Standard off-the-shelf servo motors and brackets can easily be used for these legs. A hole is made in the base for the camera on the back of the phone; a small periscope can be mounted over the hole so that the camera can be used for navigation. The design of the platform is a tradeoff between simplicity and naturalistic embodiment.

\subsection{Robot System Level Design}

Smartphones already have the advantage of providing a powerful on-board computer with an array of features that keep the robot size compact and lightweight. However, the electrical components that will bring the robot to life and help keep the smartphone in control of the robots movements must also be minimal to insure both cost and weight are low. Our design connects the smartphone to the robot platform via a simple interface using only an interface board, some server motors for the legs, and a couple of power supplies. The complete system diagram is shown in Figure 3.5. The smartphone is on the left and contributes computation, display and sensing. There are eight motors (two for each leg) connected to the smartphone through the interface. A second power supply is needed for the motors. The interface needs to drive the eight motors independently and receive commands from the phone. A walking robot will need to collect as much real time information as possible to maintain balance and quickly react to environment changes. Hence, to maintain a low latency connection and not be interrupted by radio interference, the smartphone should be directly connected to the interface. Smartphones typically have a micro-USB or similar port that can be used for this. The implementation section describes how we were able to use an off-the-shelf interface to meet all of these needs. 


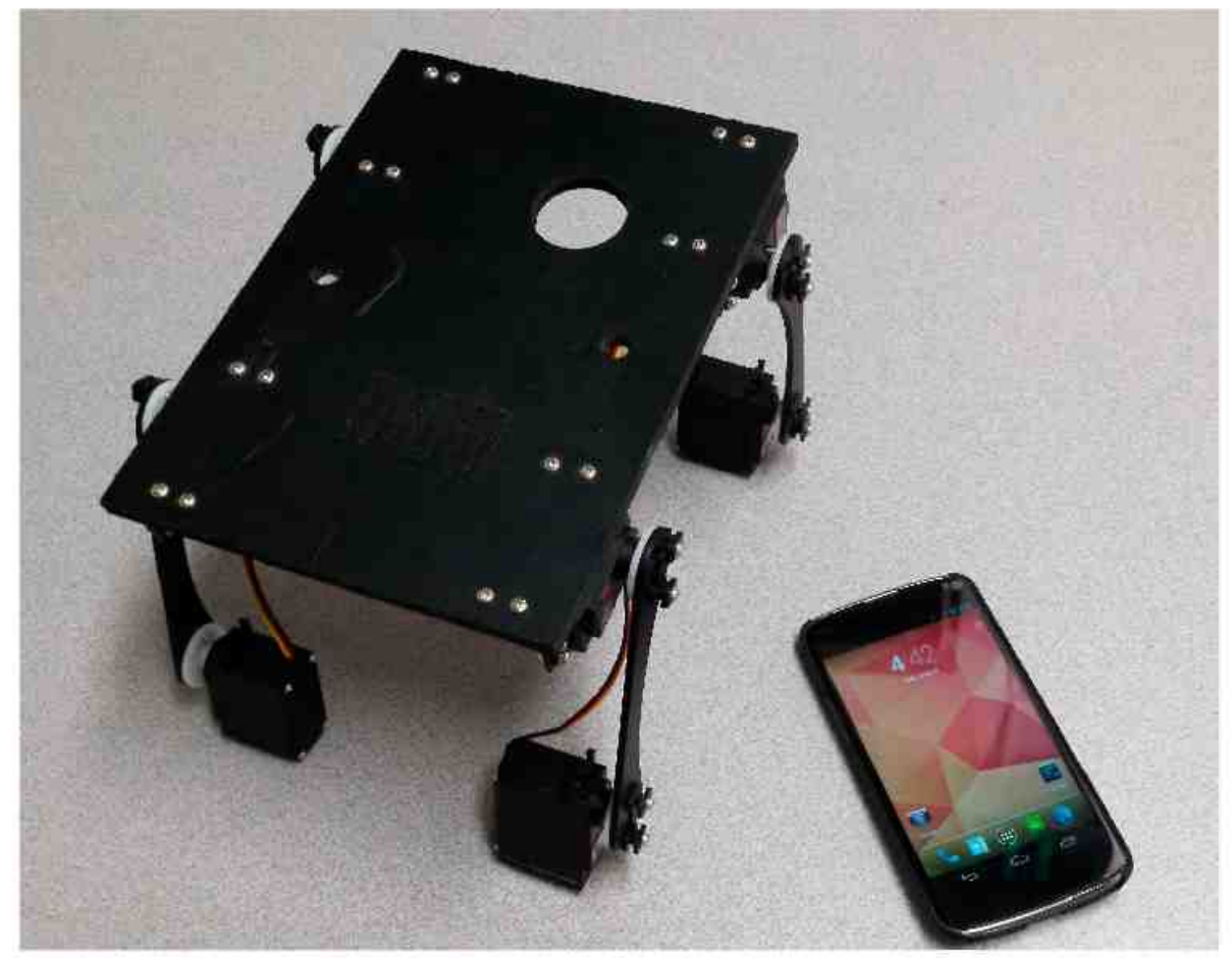

(a) Assembled base of the robot.

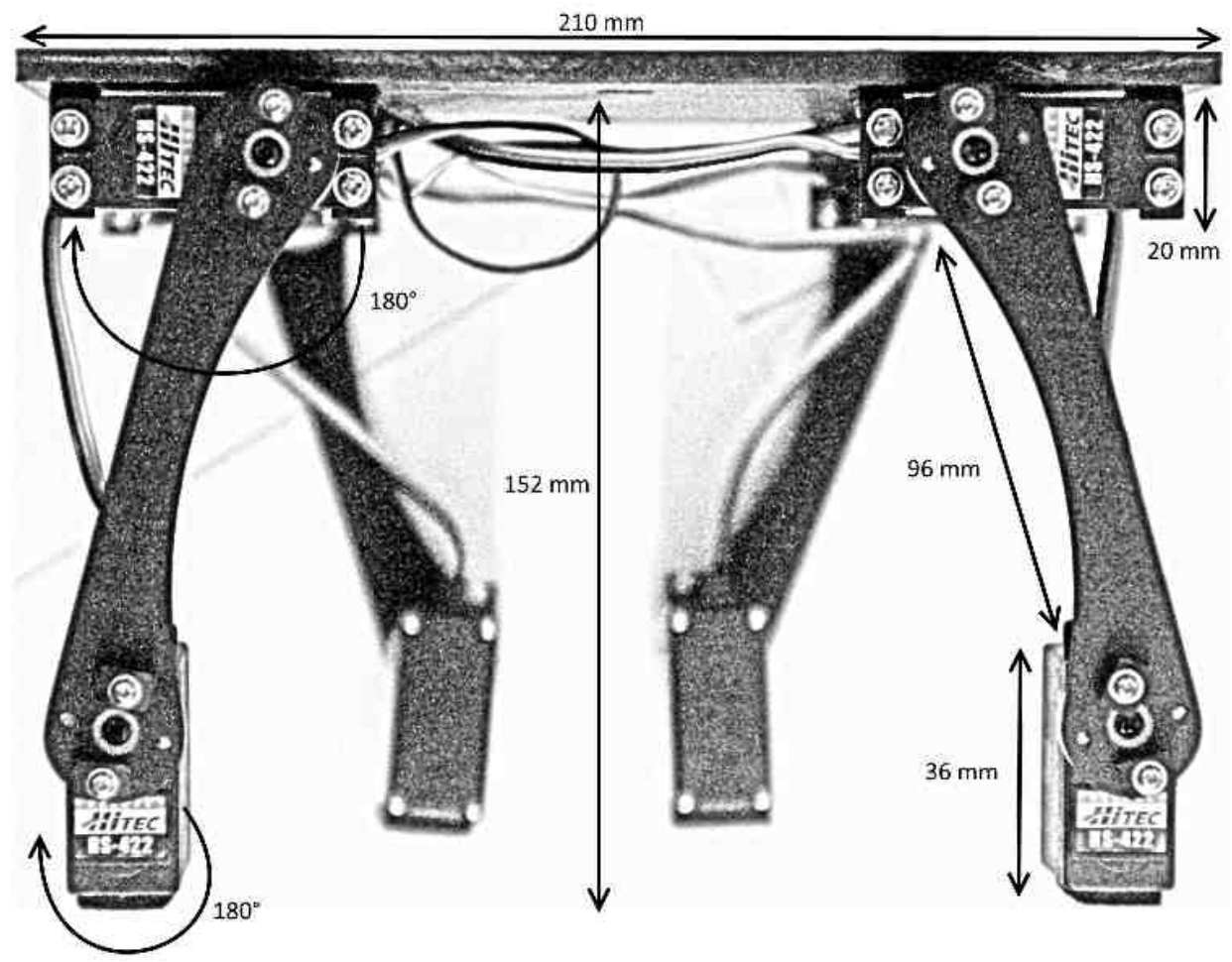

(b) Side view of the robot legs.

Figure 3.4: The base and legs of the robot platform. 


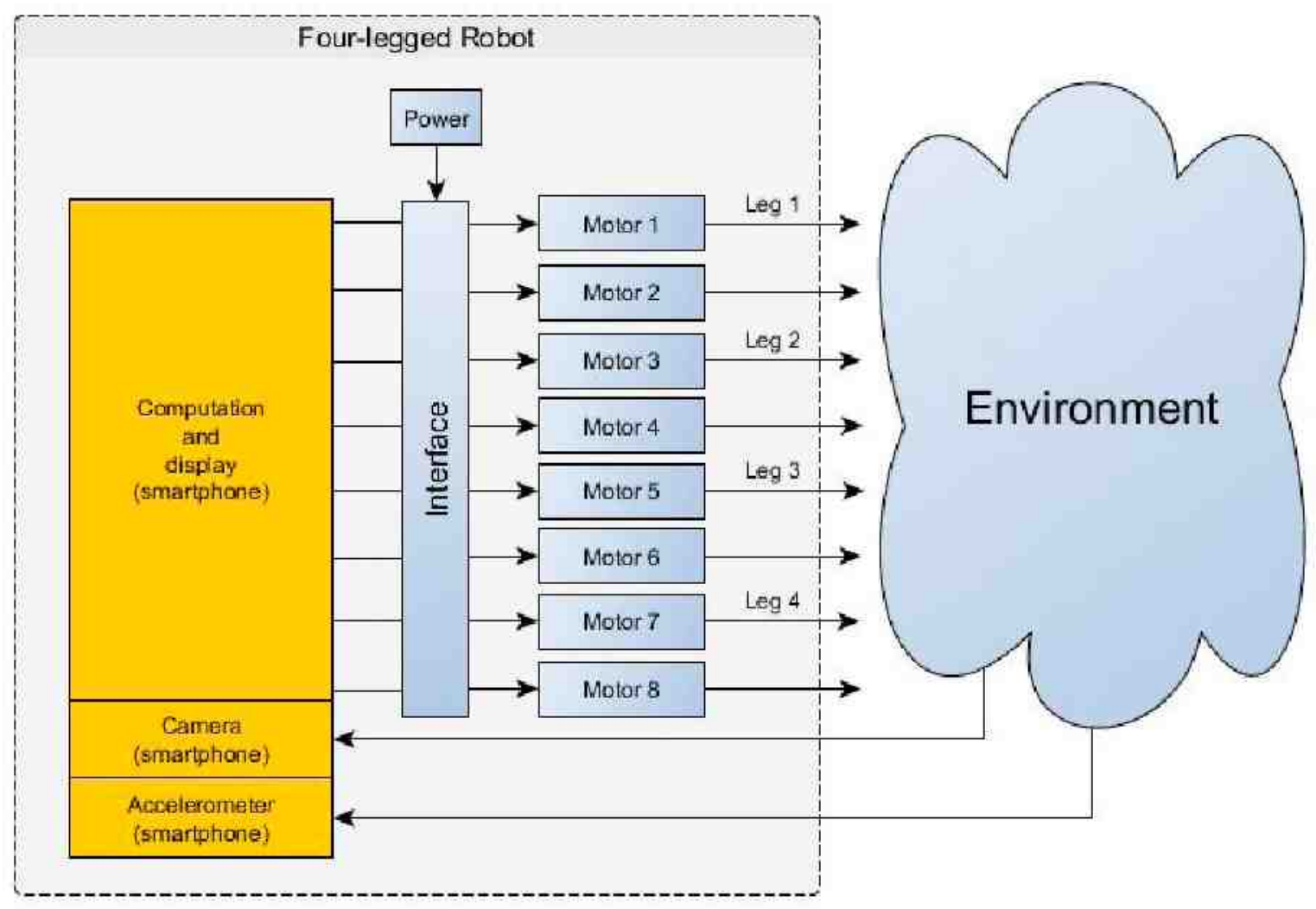

Figure 3.5: System block diagram for the robot platform.

\subsection{Robot Software Design}

The application should handle all of the robots actions and calculations and require minimal human interaction, for example tapping confirmation buttons and entering information. Figure 3.6 shows the state transitions during the execution of the application developed. All of the action states, such as walking, listening, tapping, eventually return back to the standing posture so that the user knows the robot is ready to recieve its next command. Figure 3.7 shows how the application process should run in a continuous loop until it hits an interruption (e.g. the robot falling over). The smartphone will processes commands from an automated process or an event from the environment and send it to the servo controller. At the same time it will determine if the robot is stable and either continue to process commands or suspend commands until the robot is back to the stable state. Events from the environment can 
include either a voice command or some type of image recognition like facial expressions or object detection.

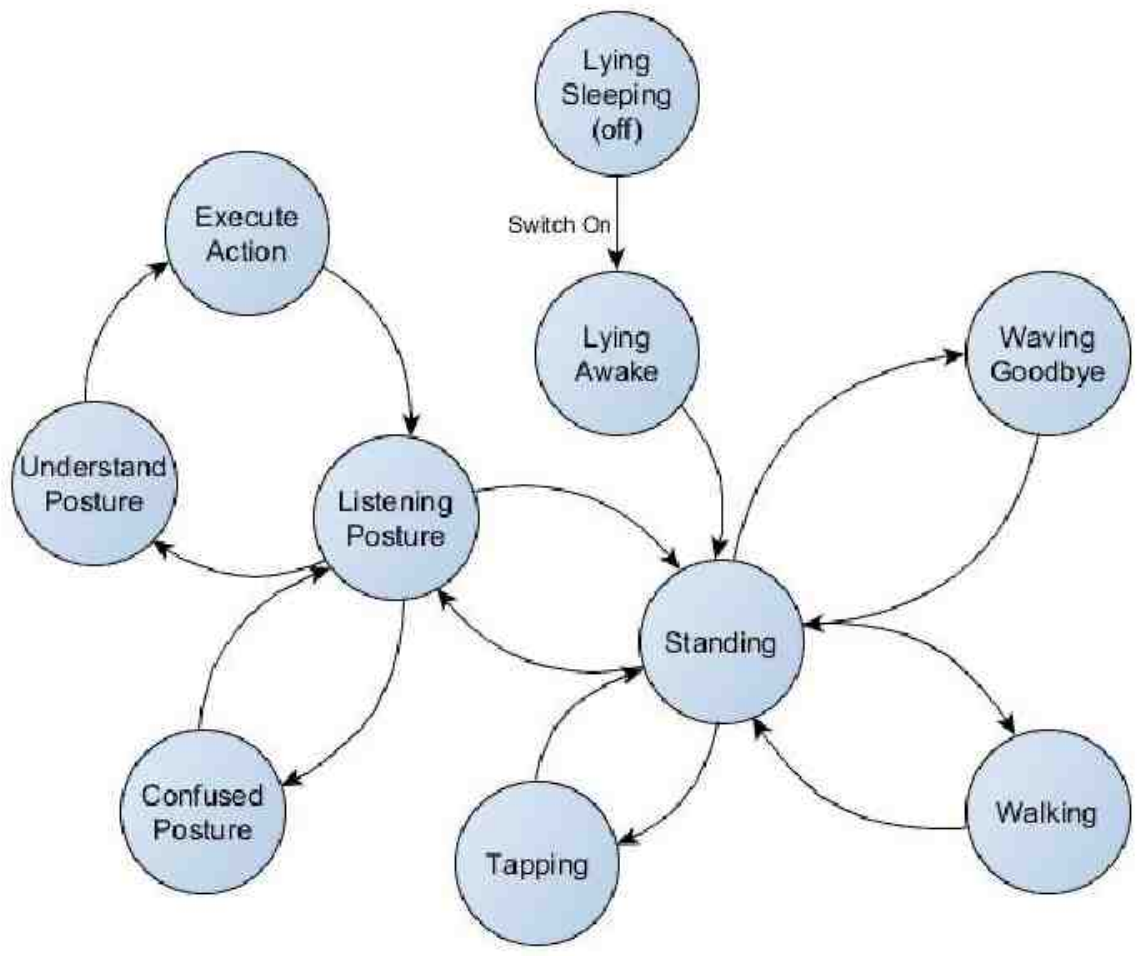

Figure 3.6: State transition diagram for robot application.

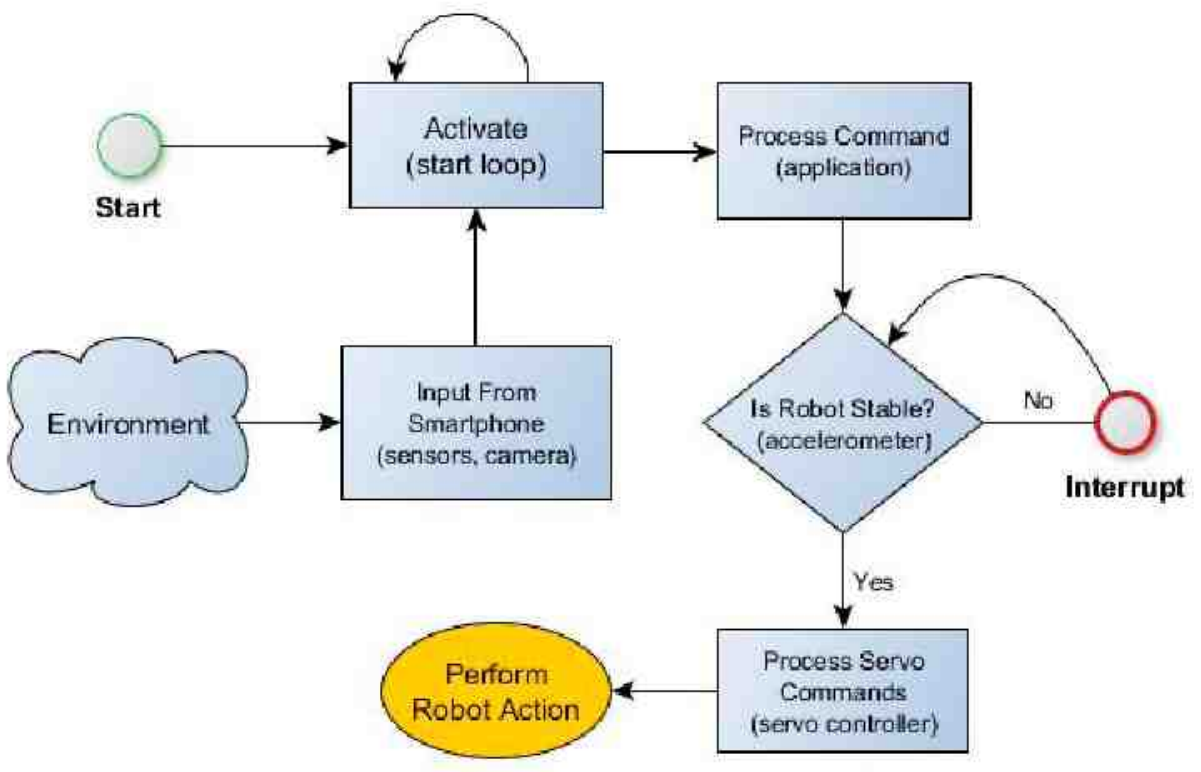

Figure 3.7: Execution proccess for robot application. 


\section{Implementation}

\subsection{Hardware}

The final implementation of our robot can be seen in Figure 4.1. Besides the smartphone and a servo controller, other parts had to be purchased. For example, we positioned the Nexus 4 to lay flat on our board to make sure our robot wasn't top-heavy while walking but there was no way for the camera to see forward. Fortunately, a company called Photojojo provides a Smartphone Spy Lens that is like a periscope enabling our robot to still see forward without propping the smartphone upright. Usually infrared sensors are used to determine the distance of an object and provide a simple object avoidance system but the main drawback to using an infrared sensor is the reliability when the robot is outside in direct sunlight. We think having the camera available for our vision system will allow more flexibility than an infrared sensor especially for object detection. A Phidgets [12] server controller board was mounted horizontally on top of the smartphone with a 9-volt battery to provide the circuit board with power. Figure 4.2 shows the schematic of everything connected in our walking robot.

A smartphone has the advantage of providing a powerful on-board computer with an array of features while keeping the robot size compact and lightweight. The smartphone used in this project is the Android-based LG Google Nexus 4. It has a $1.512 \mathrm{GHz}$ quadcore Krait CPU, 2 GB of RAM, a 3-axis accelerometer, an 8-megapixel camera and several other sensors. These are typical features for many smartphones and they are well-suited for robotics applications. Most smartphones at least support the USB 2.0 standard that can $\mathrm{read} /$ write on an average speed greater than 30MBps. Some Android-based devices do not 


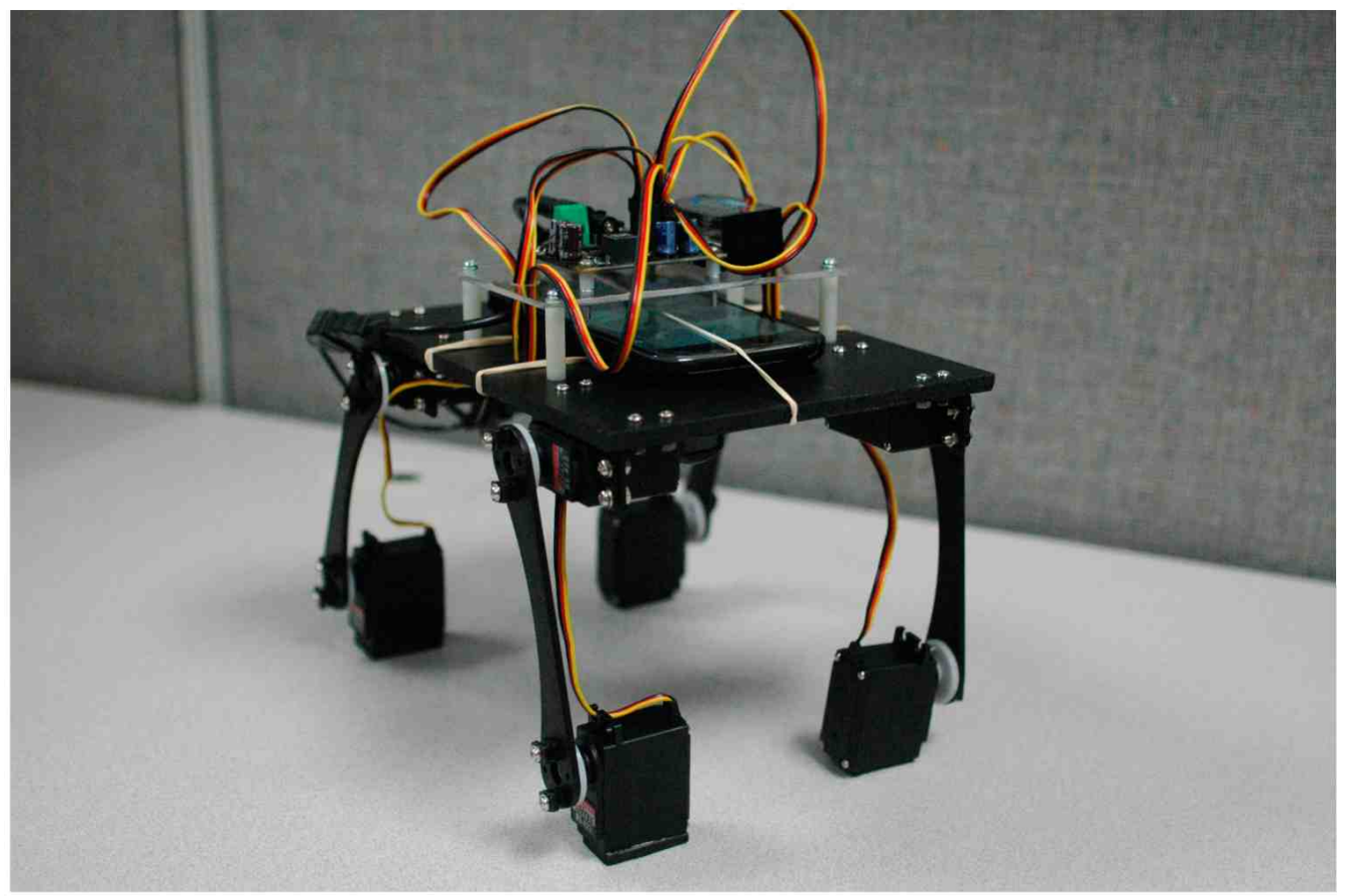

(a) Walking robot assembled

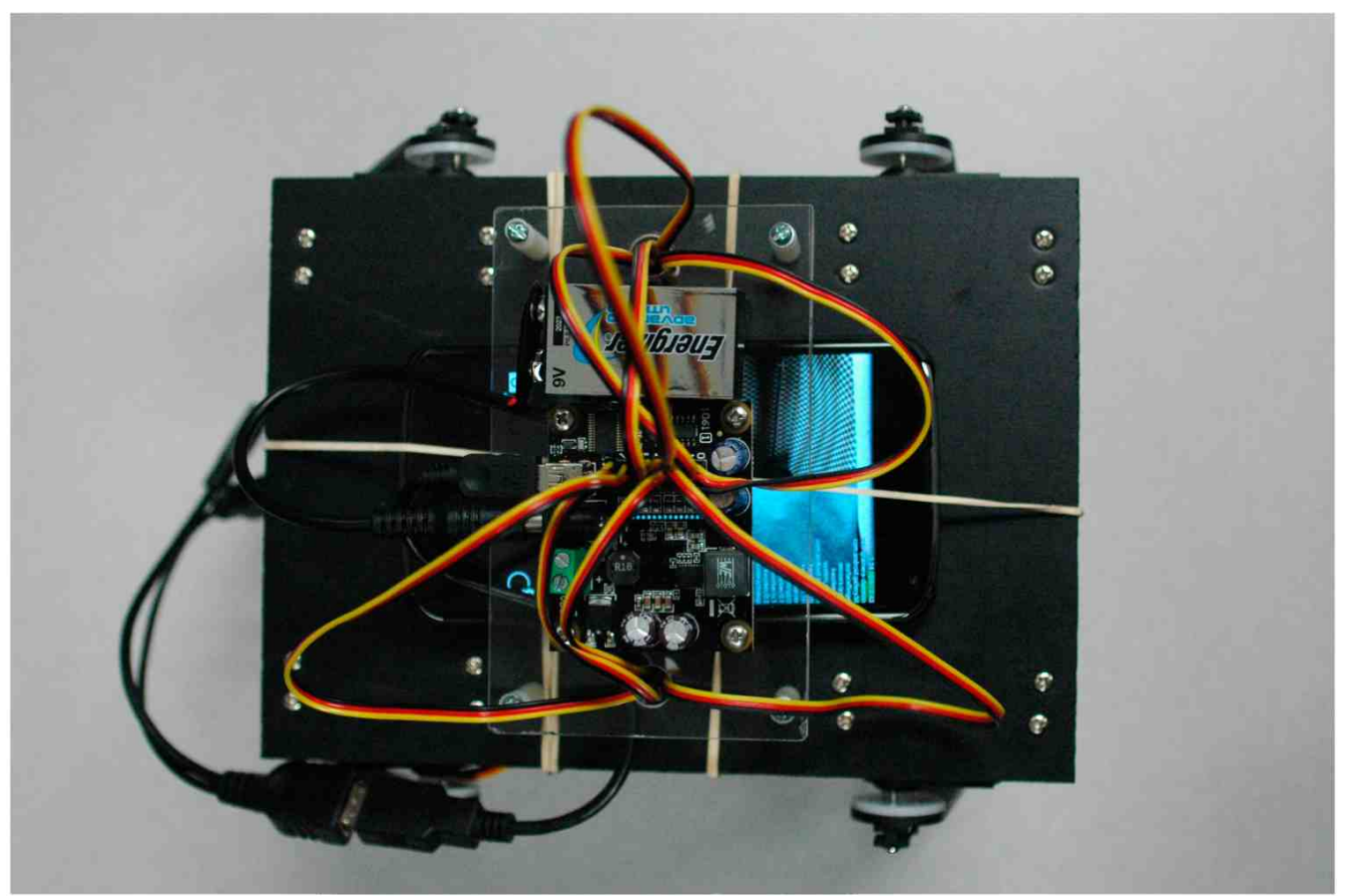

(b) Top view of robot

Figure 4.1: Final construction of smartphone walking robot. 


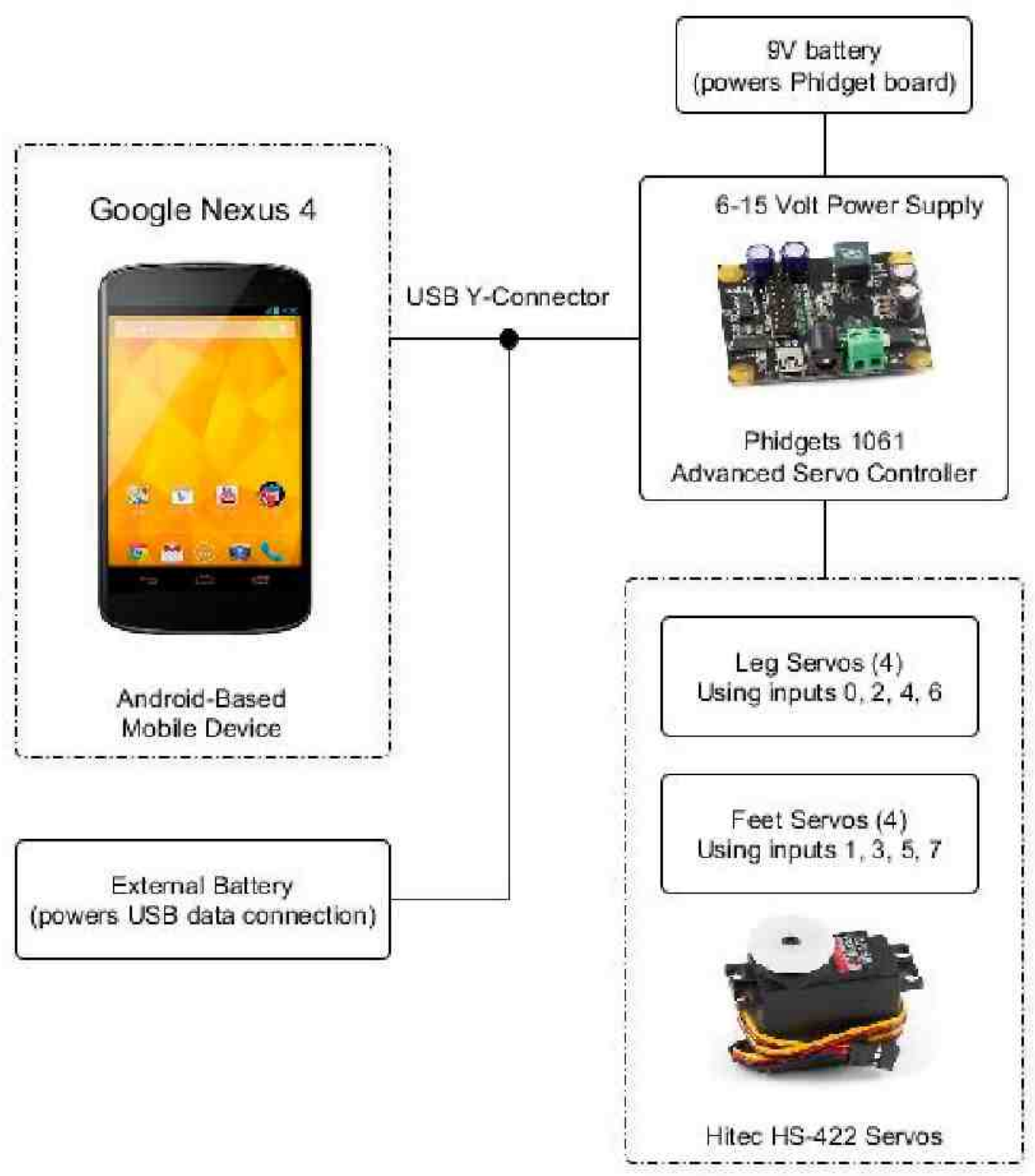

Figure 4.2: Schematic showing the connections for each robot component.

provide the correct voltage required to power USB devices like a keyboard or mouse. This seems to be a rare case and unfortunately a 'hack' to the Android kernel and a three-way Y cable connecting a USB power pack is usually required to enable USB support.

The Phidgets Advanced Servo Controller 1061 is a motor controller that uses a mini-USB cable connection to control the position, acceleration, and velocity of up to 8 servo motors in a compact module that requires 6-15 volts to power. The circuit board can be purchased 
individually or in a kit that comes with 4 Hitec HS-422 Deluxe servo motors and a power supply. Specifications state that each servo is powered at up to 3.4 amps using a switching regulator that protects the motors from over-voltage and maintains a high resolution of 125 steps per degree. The position of each servo is controlled by the smartphone using an Android application and a library of functions provided by Phidgets. Also included with the installation of the drivers and libraries is the Phidget Control Panel which can help test your Phidget board to make sure it is working properly, calibrate servos, and update the firmware. At the moment, direct control through a cable is not available for $\mathrm{iOS}$ devices. Until it is supported, the Phidgets library includes a WebService that broadcasts all events and data from a Phidget board over a network. Phidgets also carries a range of I/O boards that can be connected to various sensors but for this project we want to minimize our payload and rely on the built-in sensors provided by the smartphone.

\subsection{Software}

The Android mobile platform provides all the tools for creating applications that take full advantage of the hardware capabilities for each device. The software development kit (SDK) allows programmers to develop Android software in Java or a native development kit (NDK) is available for $\mathrm{C} / \mathrm{C}++$ developers that strive to increase application performance. Using either the Eclipse IDE with the Android Development Tools or Google's early preview of the Android Studio development environment, implementation of a native Android application to a device is pretty straightforward. Android is also an open source operating system which makes it possible for developers to modify the Linux kernel for even more customization.

The software stack for the robot is shown in Figure 4.3. At the bottom is the Android operating system which is open source, making it possible for developers to modify the kernel, as mentioned in Section 3.3, to enable USB support on the Nexus 4. It provides all of the tools needed to create applications that take full advantage of the hardware capabilities for each device. Using Android Studio, we developed a small Java application that can run 
on any device with Android Honeycomb 3.1 or higher. Source code for this application can be found in Appendix A. The application has a user interface on the phone that displays simple controls to move the servos and show accelerometer readings for testing purposes through the Phidgets Library which supports a variety of programming languages. You can access an ample amount of API documentation for each language with its own libraries, code examples and drivers at the Phidgets website.

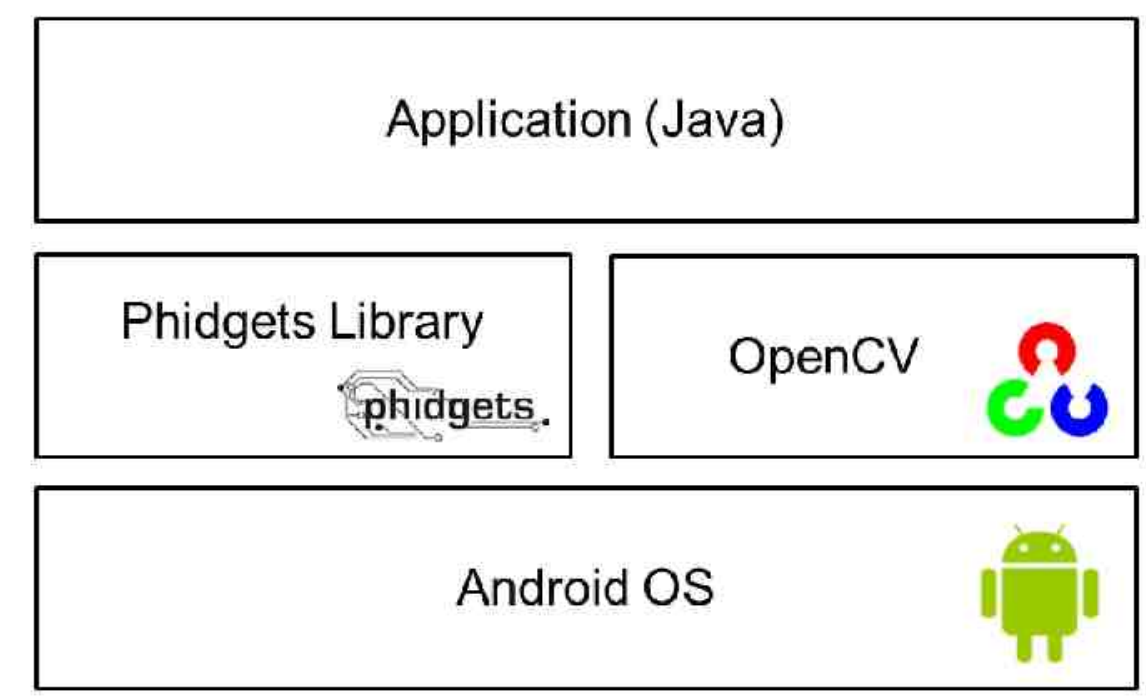

Figure 4.3: Software stack for the robot platform.

In addition to this we used the OpenCV computer vision library to test the camera and used some simple machine vision algorithms for navigation. OpenCV provides many builtin functions such as image transformations and object detection. For instance, the image generated by the camera is upside down but OpenCV helps implement a simple remapping routine that reflects the image along the x-axis. For the final implementation the total cost of materials turned out to be around $\$ 250$ (USD) without the cost of the smartphone (See Table 4.1). Older unlocked phones can be purchased on websites such as eBay for around $\$ 100$ and usually have suitable specifications for most projects. 


\begin{tabular}{|l|l|}
\hline Google Nexus 4 & $\$ 200$ \\
\hline Phidget Advance Servo Kit (4-motors) & $\$ 140$ \\
\hline Hitec HS-422 Deluxe Servo (4) & $\$ 50$ \\
\hline Lynxmotion Femur for legs (4) & $\$ 25$ \\
\hline Photojojo Smartphone Spy Lens & $\$ 25$ \\
\hline Stiff board for base & $\$ 10$ \\
\hline TOTAL COST(USD) & $\$ 450$ \\
\hline
\end{tabular}

Table 4.1: Prices paid (USD) for robot construction parts.

\subsection{Walking Technique}

As an example of how easy it is to program, the walking was mostly accomplished through observation and trial-and-error. Our first attempt at walking was having the robot push off its hind legs like a frog but the movement would jostle the smartphone and circuit board, and then eventually offset the balance of the robot causing it to fall over. Walking needed to either have one leg move at a time so that there are three contact points to keep the robot stable or alternate a pair of legs similar to the movement of a dog like in Figure 4.4(a) [21]. The approach we chose involved alternating the legs even though this also makes the robot less stable than if we decided to move one leg at a time, but it has the advantage of walking more efficiently and faster. Using the 4 servos to control the joints where our legs are attached and the addition of 4 more servos as knee joints/feet propelled the robot forward while maintaining balance. For traction, rubber foam stickers were placed on the bottoms of the servos at the contact points.

The accelerometer sensor is also used to detect problems when it is turned on during a calibration state or while it is walking. For instance, if there seems to be a malfunction with a leg or the robot falls over, the accelerometer is able to alert the robot to stop walking and/or be programmed to alert a user via wireless communication such as text. Initially the 


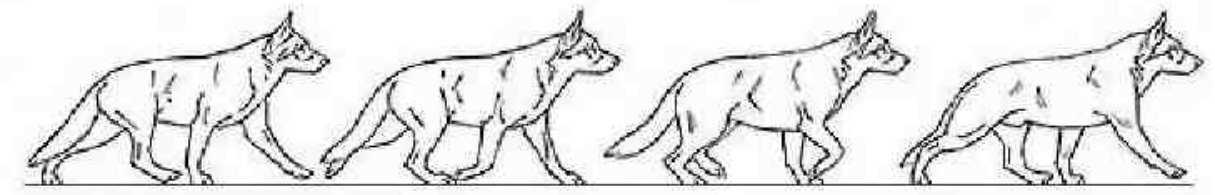

(a) A 'supported trot' always has contact with the ground for support but can still move at a decent speed [22].

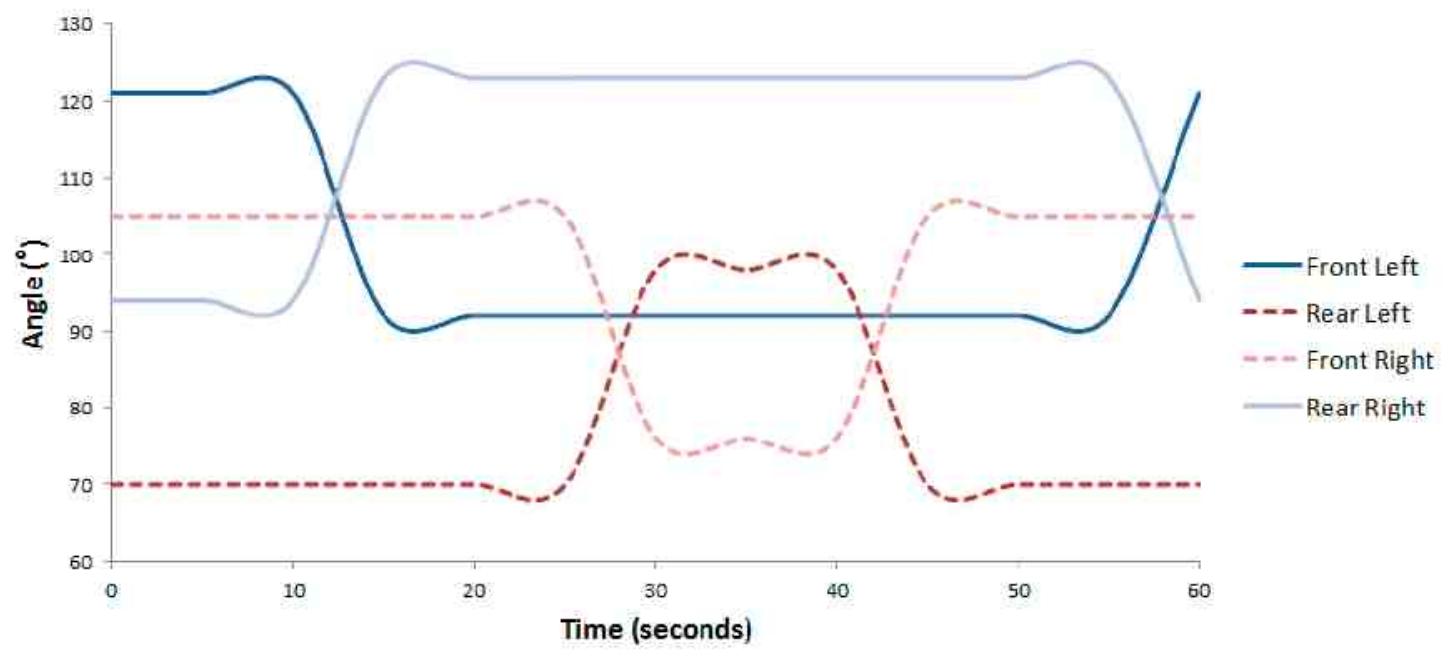

(b) Angles ploted for all four robot legs as it walks. Two legs diagonally opposite from each other alternate movement giving the robot a brisk walk with stability.

Figure 4.4: Walking gait of a dog (a) was used as a model for robot leg movement (b). 
accelerometer sensor was going to be used to keep the robot balanced dynamically but since we designed a four-legged robot instead of an upright two-legged robot, the functionality of the accelerometer diminished. 


\section{Experiments and Results}

So far we have created two demonstrations of the robot's social capabilities as described in the following two subsections. The first is a simple demonstration of the robot's ability to move about our environment and physically interact with us. The second is an exposition of a sequence of body postures designed to enhance conversations with humans.

\subsection{Good Morning Robot!}

The first demonstration involves the smartphone robot playing the role of a personal assistant. The robot is 'sleeping' on the bed and awakens when the smartphone's alarm rings. It proceeds to walk over the bed to tap the arm of its boss and announce any schedule commitments. This gives us a hint at the possibilities of having a robot assistant that has all of the information of a smartphone coupled with a familiar and capable four-legged body. The link

to the video for this demonstration is available in Figure 5.1. We have presented this video at seminars and talks and it has been well received without any mention of the creepy feelings that accompany some artificial moving creatures as in the so-called uncanny valley effect.

\subsection{Robot Body Postures for Conversation}

The second demonstration consisted of having the robot assume some set postures that would be intended to communicate something conversational to a human. This type of body language would enhance typical verbal or visual messages, making the robot seem more lifelike. 


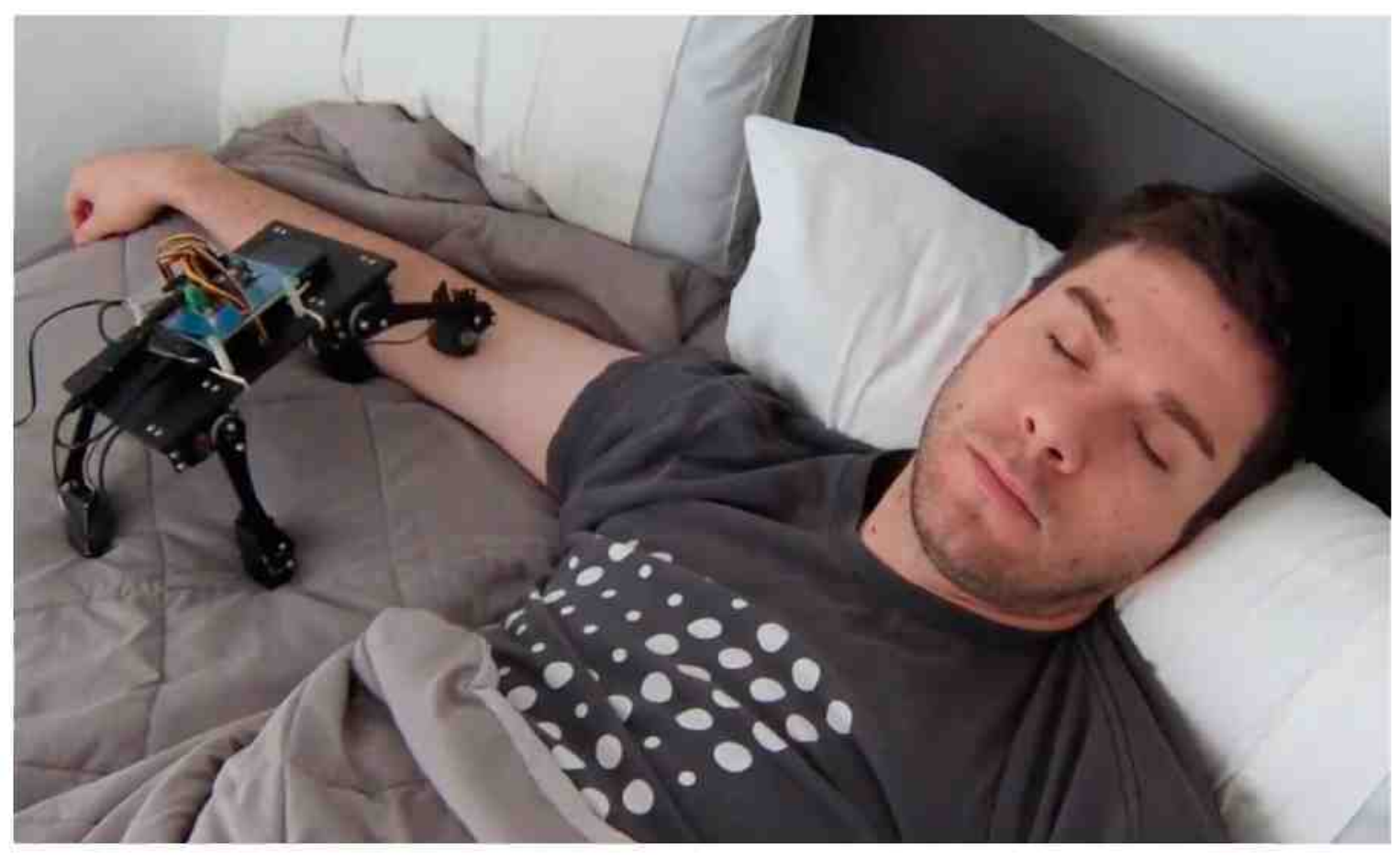

Figure 5.1: https: $/$ iwww youtube $\operatorname{com}^{i}$ watch $? \mathrm{v}=$ ErMmKLIDkp4

Figure 5.2 shows an example of two such postures with the accompanying facial expressions. A video sequence of our four postures was created in order to survey a typical user's interpretation of each posture without the accompanying face. The video is available here:

\section{https:/iwww youtube com ${ }^{\prime}$ watch? $\mathrm{v}=\mathrm{PtCIbGjJV4c}$}

The survey questionnaire was as follows:

You are interacting with the robot from the video. What do you think it is trying to convey after each posture? Please put the posture number with the interaction that you think matches:
a) It is confused
b) It is ready to listen
c) It understands what you said
d) It is about to leave
e) Don't know 


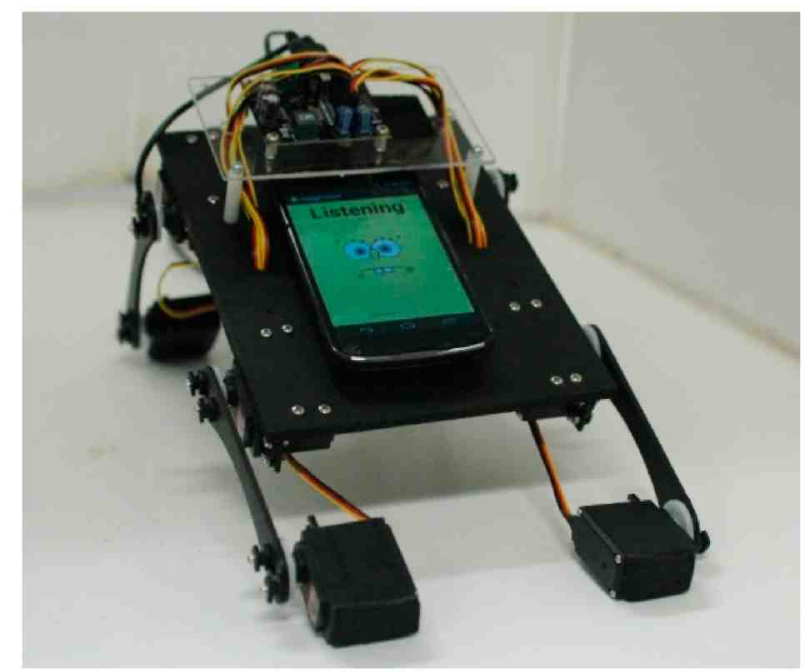

(a) ready to listen

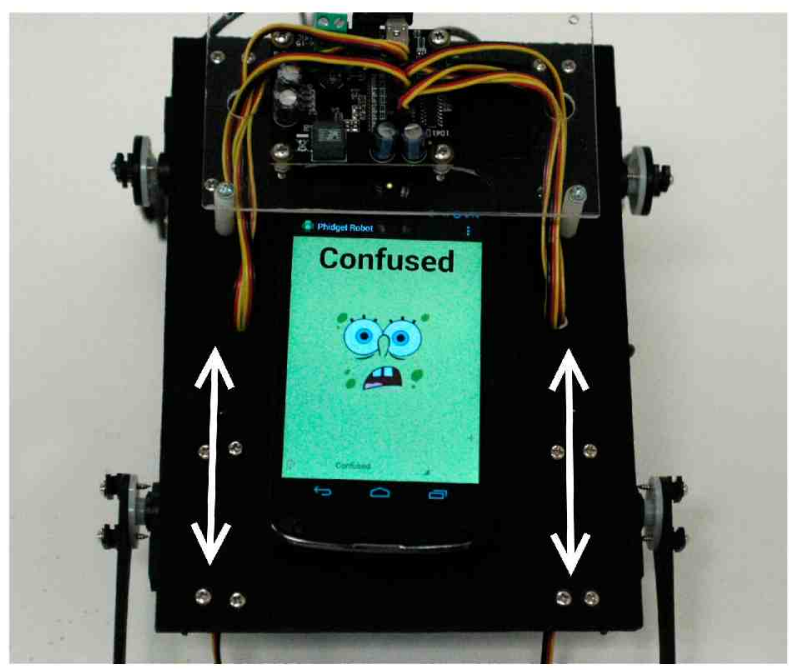

(c) confused

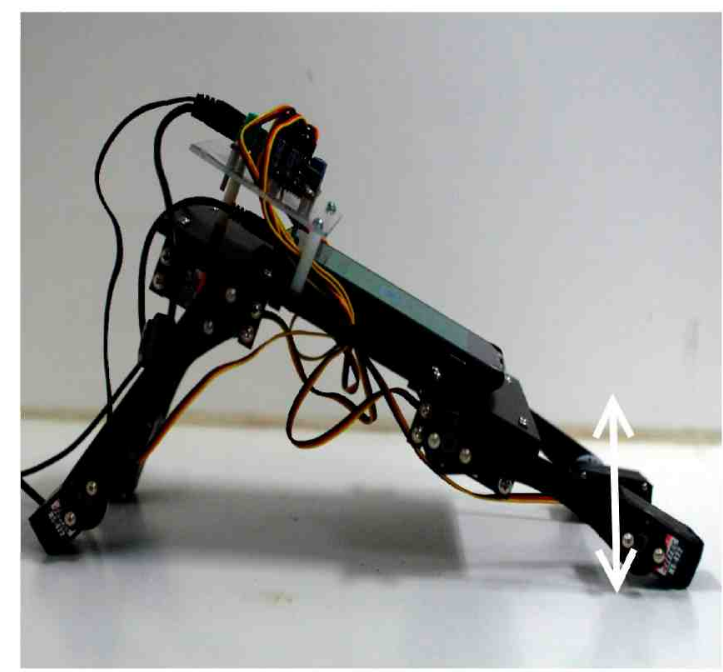

(b) understood

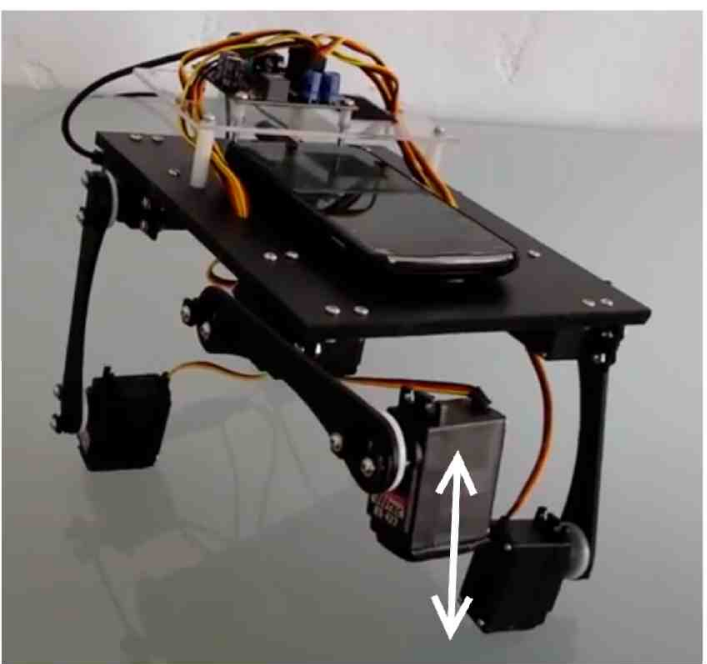

(d) waving goodbye, leaving

Figure 5.2: Example postures (a) bowing action as if listening, (b) front of robot nods if a command is understood, (c) robot rocks forward and backwards if confused, and (d) waves front arm waving goodbye. 
We did a small, informal study of ten participants gathered from faculty and staff close to our offices. The results of the survey are shown in Figure 5.3. The first two postures were accurately interpreted by most people in the survey. The last two had some problems but this was attributable to their ambiguous nature when not combined with verbal and facial cues. A full video with facial expressions is here:

https:/ $i$ www youtube com $i$ watch?v=B43FYX4GtXs

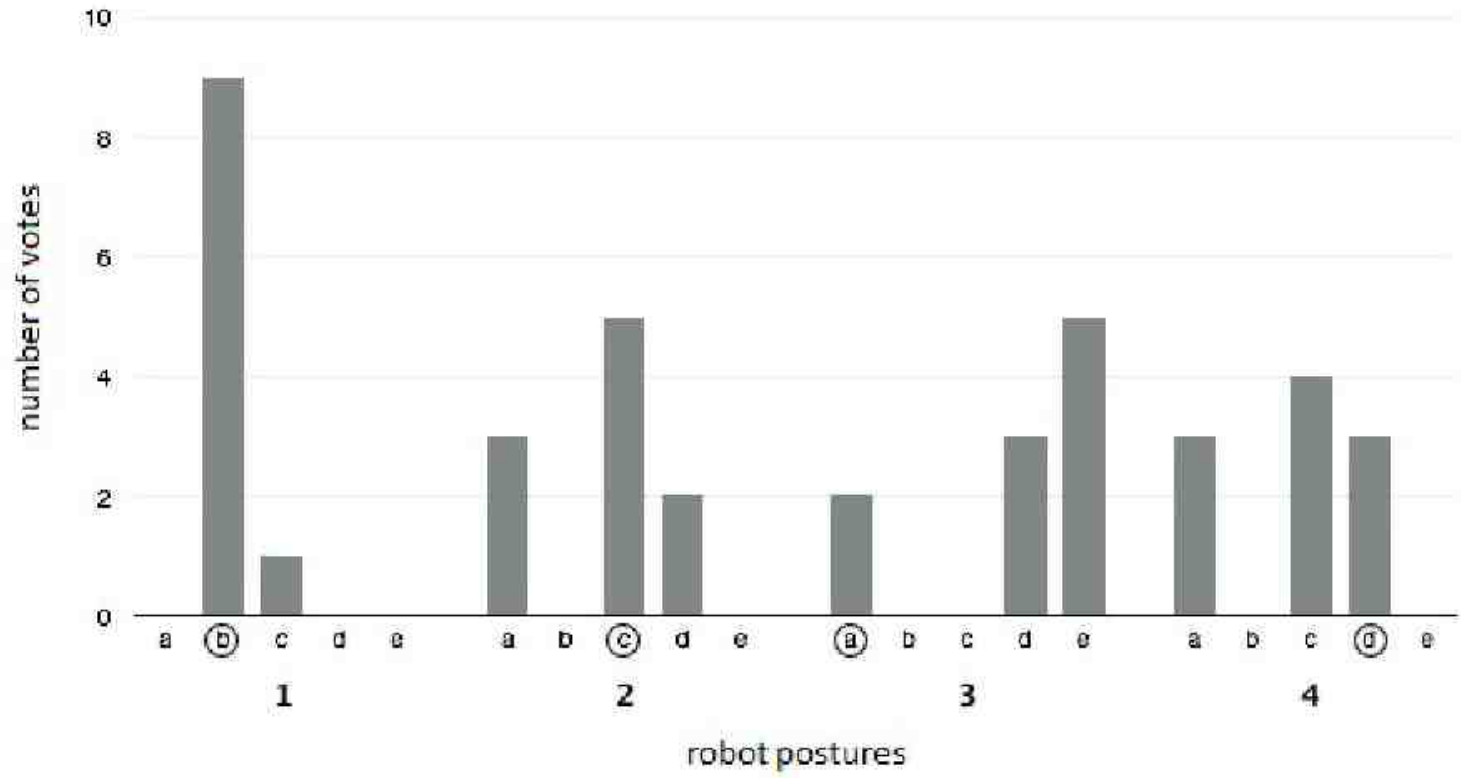

Figure 53: Survey results for robot postures (intended response is circled for each posture). Posture 1 is 'ready to listen', posture 2 is 'understand', posture 3 is 'confused', and posture 4 is 'waving goodbye'.

The survey participants also viewed the robot postures with the facial expressions and all agreed that the combination was clear and added to the feeling of having a real conversation. 


\section{Conclusion}

\subsection{Summary}

A simple four-legged robot platform that can transform a smartphone or tablet into an autonomous, walking social robot has been presented. Simple walking and posturing have been demonstrated to show how such a robot can better play the role of companion or assistant in the office or home. The platform offers students and researchers an inexpensive development system to explore social robotics. Our preliminary tests have shown that the system is easy to program and is well received by those interacting with it. The open nature of the software makes it possible to develop low-cost, downloadable apps by anyone who wishes to add to the system and customize it.

\subsection{Future Work}

At first, we planned to build a biped platform that can use the smartphone's accelerometer and gyroscope to keep balance when standing and walking upright and this might be explored again now that we have a working platform in place. We hope that this will give our robot a more human-like posture so that users will feel more immersed while interacting with the robot. We also hope to develop a better vision system that can help to achieve richer interactions in which the robot can interpret human gestures and emotions. This type of software is also openly available and should only have to be ported and adjusted to fit the platform. 
Incorporating some type of voice activation with the Google Voice API or creating a mobile application that allows the user to connect to the robot via the mobile network would allow the user to constantly monitor the robot for data reports or errors. This might be valuable for a parent who could use the robot as a companion to monitor a young child in the other room. The robot could alert the parent when the child is crying or out of sight. Additionally it could interact with the child by playing games and providing some educational content. This would also be beneficial if a project required the constant monitoring of a swarm of robots that are programmed to perform a task in a commercial environment.

Cloud computing is becoming an increasingly popular term and we hope to someday leverage the benefits it has to offer. Currently, researchers are looking at the idea of robots that could use cloud computing to download information on an object it has never seen before or learn a new task that it was not originally programmed for [23]. With this platform in place, there is no need for the user to download software updates or patches since they can be pushed to the cloud and the robot could download them at a convenient time. The robot could also use the remote servers to perform CPU-heavy computations which could speed up robot actions and save battery life. Overall, there are many similar possibilities and we look forward to experimenting with the robot and working with others who are also interested. 


\section{References}

[1] http://www.romotive.com/

[2] http://www.overdriverobotics.com/

[3] Vincent, J., "Is the mobile phone a personalized social robot?" in Intervalla Vol. 1, 2013, http://www.fc.edu/intervalla/images/pdf/6_vincent.pdf, 2013.

[4] Wainer J, Feil-Seifer DJ, Shell DA, Mataric MJ, "The role of physical embodiment in human-robot interaction" in IEEE proceedings of the international workshop on robot and human interactive communication, Hatfield, UK, pp 117-122, 2006.

[5] Wainer J, Feil-Seifer D, Shell D, Mataric M, "Embodiment and human-robot interaction: A task-based perspective", pp 872-877, 2007.

[6] Jung Y, Lee KM "Effects of physical embodiment on social presence of social robots. Presence 2004", Spain, pp 8087, 2004.

[7] Adalgeirsson, S. and Breazeal, C., "MeBot: A robotic platform for socially embodied telepresence", Proc. HRI 2010, ACM Press, 15-22.

[8] N. Oros, J. Krichmar, "Smartphone Based Robotics: Powerful, Flexible and Inexpensive Robots for Hobbyist, Educators, Students and Researchers" in CECS Technical Report $13-16,2013$.

[9] N. Herget, W. Keyes, C. Wang, "Smartphone-based Mobile Robot Navigation”, 2012 [Online].

[10] S. Gobel, R. Jubeh, S. Raesch, A. Zundorf, "Using the Android Platform to control Robots" in Proc. of the Robotics in Education Conference, 2011.

[11] Raibert, M. et al., "Bigdog, the rough-terrain quadruped robot" in Proceedings of the 17th World Congress The International Federation of Automatic Control (IFAC), 2008.

[12] http://www.phidgets.com

[13] MIT Leg Laboratory, http://www.ai.mit.edu/projects/leglab/, 1999.

[14] Breazeal, C., "Cynthia Breazeal: The rise of personal robots" in TED Talks, http://www.ted.com/talks/cynthia_breazeal_the_rise_of_personal_robots, 2011.

[15] http://www.rethinkrobotics.com/

[16] http://www.apple.com/ios/siri/

[17] http://www.google.com/landing/now/ 
[18] http://www.romela.org/main/Robots

[19] http://www.ai.mit.edu/projects/leglab/robots/3D_hopper/3D_hopper.html

[20] LeBouthillier, A. "W. Grey Walter and his Turtle Robots", in The Robot Builder, 1999, http://www.rssc.org/content/w-grey-walter-and-his-turtle-robots, 2010.

[21] Nunamaker, D. Blauner P. "Normal And Abnormal Gait", http://cal.vet.upenn.edu/projects/saortho/chapter_91/91mast.htm, 1985.

[22] Shaw, L. "An Over View of the Gait", http://www.gsdinfo.co.uk/General Info/An Over View of the Gait.htm.

[23] Guizzo E. "Robots With Their Heads in the Clouds", in IEEE Spectrum, http://spectrum.ieee.org/robotics/humanoids/robots-with-their-heads-in-the-clouds, 2011.

[24] Brooks, Rodney A. "Intelligence without representation", Artificial Intelligence 47 (13) (1991), 139-59.

[25] https://www.sparkfun.com/products/11343 